\title{
On the Lundgren-Townsend model of turbulent fine scales
}

\author{
D. I. Pullin \\ Graduate Aeronautical Laboratories 105-50, California Institute of Technology, Pasadena, \\ California 91125
}

P. G. Saffman

Applied Mathematics 217-50, California Institute of Technology, Pasadena, California 91125

(Received 14 May 1992; accepted 24 August 1992)

\begin{abstract}
The strained-spiral vortex model of turbulent fines scales given by Lundgren [Phys. Fluids 25, 2193 (1982)] is used to calculate vorticity and velocity-derivative moments for homogeneous isotropic turbulence. A specific form of the relaxing spiral vortex is proposed modeled by a rolling-up vortex layer embedded in a background containing opposite signed vorticity and with zero total circulation at infinity. The numerical values of two dimensionless groups are fixed in order to give a Kolmogorov constant and skewness which are within the range of experiment. This gives the result that the ratio of the ensemble average hyperskewness $S_{2 p+1} \equiv \overline{(\partial u / \partial x)^{2 p+1}} /\left[\overline{(\partial u / \partial x)^{2}}\right]^{(2 p+1) / 2}$ to the hyperflatness $F_{2 p} \equiv \overline{(\partial u / \partial x)^{2 p}} /\left[\overline{(\partial u / \partial x)^{2}}\right]^{p}, p=2,3, \ldots$, is constant independent of Taylor-Reynolds number $R_{\lambda}$, as is the ratio of the $2 p$ th moment of one component of the vorticity $\Omega_{2 p} \equiv \overline{\overline{\omega_{x}^{2 p}}} /\left(\overline{\overline{\omega_{x}^{2}}}\right)^{p}$ to $F_{2 p}$. A cutoff in a relevant time integration is then used to eliminate vortexsheet-induced divergences in the integrals corresponding to $\overline{\overline{\omega_{x}^{2 p}}}, p=2,3, \ldots$, and an assumption is made that the lateral scale of the spiral vortex in the model is the geometric mean of the Taylor and the Kolmogorov microscales. This gives $\Omega_{2 p}=\hat{\Omega}_{2 p} R_{\lambda}^{p / 2-3 / 4}$, $F_{2 p}=\hat{F}_{2 p} R_{\lambda}^{p / 2-3 / 4}$ and $S_{2 p+1}=\hat{S}_{2 p+1} R_{\lambda}^{p / 2-3 / 4}, p=2,3$,..., with explicit calculation of the numbers $\hat{\Omega}_{2 p}, \hat{F}_{2 p}$, and $\hat{S}_{2 p+1}$. The results of the model are compared with experimcntal compilation of Van Atta and Antonia [Phys. Fluids 23, $252(1980)$ ] for $F_{4}$ and with the isotropic turbulence calculations of Kerr [J. Fluid Mech. 153, 31 (1985)] and of Vincent and Meneguzzi [J. Fluid Mech. 225, 1 (1991)].
\end{abstract}

\section{INTRODUCTION}

A comprehensive theory of turbulent fine scales has been actively sought by researchers for many decades. Such a theory would need to explain many quantitative and qualitative features of turbulent flows observed in both experiment and in numerical simulation while containing sufficient predictive capability to enable decisive testing by future measurement and calculation. Reasonable minimum requirements for such a theory or model would seem to be the provision of a basis, preferably dynamical, for understanding Kolmogorov scaling in the inertial and dissipation ranges, and dissipation intermittency at large Reynolds numbers. More stringent requirements may refer to calculations of the one-point velocity-derivative statistics and the two-point and multipoint one-time velocity structure functions.

One approach to this problem is the use of vortexbased physical models of turbulence fine scales using spatial ensembles of small-scale structures represented by local solutions of the Navier-Stokes equations. Specific models using either tube and sheetlike structures have been proposed by Townsend, ${ }^{1}$ Corrsin, ${ }^{2}$ Tennekes, ${ }^{3}$ Saffman,,${ }^{4}$ and Lundgren. ${ }^{5}$ In general these models have sought to contain in the basic solution the essential mechanism of balance between vorticity production through amplification of the vorticity vector by the local rate-of-strain tensor in vortex layers or tubes and vorticity dissipation by viscosity. Additional features such as the spotty or intermittent nature of the dissipation can then be viewed as a result of the nonuniform spatial distribution of vorticity resulting from either the character of the local solution or the spatial separation of members of the ensemble.

Townsend ${ }^{1}$ attempted to calculate the one-dimensional energy spectrum in the dissipation range for isotropic turbulence through the use of randomly oriented collections of Burgers' tubelike and sheetlike local solutions of the Navier-Stokes equations. Sheets have often been preferred over tubes because in the Burgers vortex-tube solution [see Eq. (16)] the product of the external principal rates of strain is positive, whereas for isotropic turbulence the ensemble average of the product of the principal rates of strain is proportional to the cubic velocity derivative or skewness, which is known from measurement and simulation to be negative-in agreement with scaling arguments based on the von Kármán-Howarth equation. However, as noted by Ashurst ${ }^{6}$ and Jiménez, ${ }^{7}$ when the strain field of the vortex is itself included in the calculation, the Burgers vortex-tube solution exhibits a negative principal rates-ofstrain product over much of the vortex cross section. Ashurst finds ratios of principal rates of strain as $(-4,1,3)$ in the interior of a Burgers vortex tube with circulation $\Gamma_{0}$ equal approximately to $300 v$ ( $v$ is the kinematic viscosity) with the intermediate value aligned with the vorticity, in 
rough agreement with the strain-vorticity correlation statistics found in isotropic turbulence simulations (Ashurst et $a l^{8}$ ).

Evidence suggesting vorticity in tubelike structures in homogeneous turbulence has been provided by several numerical simulations of which we cite Kerr, ${ }^{9,10}$ Ashurst et al. ${ }^{8}$ Hosokawa and Yamamoto, ${ }^{11}$ She et al. ${ }^{12}$ Ruesch and Maxey, ${ }^{13}$ and Vincent and Meneguzzi. ${ }^{14}$ This has been summarized by Jiménez ${ }^{7}$ who, for isotropic turbulence at Taylor-Reynolds numbers $R_{\lambda}$ of order 100 , concludes that the data are consistent with the presence of compact Burgers tubelike structures with $\Gamma_{0} / v \approx 400 \pm 200$. These vortices appear to dominate the local flow and to interact only weakly with each other. On the experimental side Douady et $a l .{ }^{15}$ have produced flow visualizations which appear to show the presence of persistent tubelike structures in homogeneous turbulence. The origin of these vortices is unknown but several scenarios are possible including instability at larger scales or via the roll-up of thin vortex layers. We note, however, that there is direct numerical evidence suggesting the spontaneous formation of vortex cores from a random vorticity field in two-dimensional turbulence $\left(\mathrm{McWilliams}^{16}\right.$ ).

A tube model based on Burgers vortices may be shown to produce an energy spectrum of the form $E \propto \epsilon^{1 / 2} v^{1 / 2} k^{-1} \exp \left(-A k^{2} \eta^{2}\right)$ where $k$ is the wave number, $\epsilon$ is the dissipation, $\eta=\left(v^{3} / \epsilon\right)^{1 / 4}$ is the Kolmogorov scale, and $A$ is a dimensionless constant. This contains no $k^{-5 / 3}$ range. Lundgren ${ }^{5}$ extends the original Townsend ${ }^{1}$ model by replacing the equilibrium Burgers vortices in the ensemble by slender nearly axisymmetric spiral vortices, each embedded within an axially symmetric strain field supposedly supplied by the average interaction with other members of the ensemble. The spiral vortices have a lateral scale that exceeds the Burgers radius, which is of order $(v / a)^{1 / 2}$ where $a$ is the strain rate parallel to vortex lines. Hence if the vortex circulation is nonzero, collapse toward the Burgers limit is expected (see $\mathrm{Neu}^{17}$ and Lin and $\mathrm{Corcos}^{18}$ for a discussion of this process in the context of mixing layer dynamics). Utilizing a general, large time asymptotic solution of the Navier-Stokes equation for the relaxing spiral vortex, Lundgren shows that an ensemble of such structures shows a $k^{-5 / 3}$ range and also provides a dynamical model for the cascade via the tightening of spiral turns produced by the combined effect of differential rotation and the compressive strain. Remarkably, the $k$ dependence of the energy spectrum does not depend on the detailed structure of the spiral, the latter entering the analysis only through the spectrum prefactor. The model contains several physical parameters in addition to $v$ and $\epsilon$. Therefore the presence of a $k^{-5 / 3}$ range cannot be attributed to dimensional restrictions alone but is of a truly dynamical character.

In the present paper we seek to utilize the Lundgren ${ }^{5}$-Townsend ${ }^{1}$ model in an attempt to calculate higher-order one-point velocity derivative statistics for homogeneous isotropic turbulence. In Sec. II we discuss the mathematical and physical properties of the Lundgren spiral and review briefly the derivation of the Lundgren en- ergy spectrum. In Sec. III we propose a specific spiral model based on the roll-up of a vortex layer. It is then shown in Sec. IV that numerical values of two dimensionless groups in the model can be fixed by requiring agreement with experimental estimates of the Kolmogorov prefactor and the skewness. Since the model is based on a local solution of the Navier-Stokes equations it gives phase information of the vorticity field. In Secs. V and VI this is used to calculate high-order moments, in the form of hyperflatness factors and hyperskewness factors, of the probability distributions of the longitudinal velocity derivative $\partial u / \partial x$ and one component of the vorticity. Some calculations of low-order moments of the lateral derivative $\partial v / \partial x$ are also made. Owing to vortex-sheet-induced divergences in the vorticity field at the time origin, it is found necessary to introduce an early time cutoff in a time integration which appears in the expression for the vorticity moments. The choice of cutoff is based on an assumption that the smallest active physical length scale is proportional to the model Kolmogorov microscale. In order to introduce a Taylor-Reynolds number into the analysis the further assumption is introduced (Sec. VI D) that the initial lateral scale of the spiral vortex is the geometric mean of the Taylor microscale and the Kolmogorov microscale. A free parameter associated with the time-integration cutoff is then estimated by comparison of calculated fourth-order longitudinal velocity derivative moments (the conventional flatness factor) with experimental results summarized by Van Atta and Antonia. ${ }^{19}$ In Sec. VII we make a detailed comparison of the calculated hyperflatness and hyperskewness factors with results from the numerical simulations of Kerr ${ }^{9}$ and Vincent and Meneguzzi. ${ }^{14}$

\section{THE LUNDGREN SPIRAL-VORTEX MODEL}

\section{A. Evolution of the spiral vortex}

Let $(r, \theta, z)$ be cylindrical polar coordinates with origin at the vortex center and $z$ aligned with the vorticity. Each spiral vortex is embedded within an axially symmetric strain field with velocity field $\left(u_{r} u_{\theta}, u_{z}\right)=(-a r / 2,0, a z)$ where $a(t)$ is the uniform (in space) rate of strain. Lundgren's fundamental solution for the vortex evolution is of a rather general form for which the present model is a special case chosen in order to enable numerical calculation. For reasons to be discussed further in Sec. III our model consists of a single vortex layer that is embedded in a background of opposite signed vorticity and which is in the process of roll-up in the form a nearly axisymmetric spiral vortex. When, following Townsend, $a$ is assumed constant, Lundgren's approximate solution of the NavierStokes equations describing the vorticity $\omega=\omega(r, \theta, t)$ takes the form

$$
\begin{aligned}
\omega(r, \theta, t)= & \sum_{-\infty}^{\infty} \omega_{n}(r, t) \exp (i n \theta), \\
\omega_{n}(r, t)= & e^{a t} f(\rho) \exp [-i n \Omega(\rho) \tau \\
& \left.-v n^{2} \Lambda^{2}(\rho) \tau^{3} / 3\right], \quad n \neq 0,
\end{aligned}
$$




$$
\begin{aligned}
& \omega_{0}(r, t)=e^{a t}[f(\rho, \tau)+g(\rho, \tau)], \\
& \rho(r, t)=r e^{a t / 2}, \quad \tau(t)=\left(e^{a t}-1\right) / a,
\end{aligned}
$$

where

$$
\frac{1}{\rho} \frac{d}{d \rho}\left[\rho^{2} \Omega(\rho)\right]=f(\rho)+g(\rho),
$$

$(\rho, \tau)$ are stretched space and time variables, respectively, and $\Lambda=d \Omega / d \rho$. In (1)-(4), $v$ is the kinematic viscosity, $\exp (a t) f(\rho)$ is the $\theta$-averaged vorticity for the spiral, $\exp (a t) g(\rho)$ is the axisymmetric background vorticity, and

$$
\Gamma(r, t)=2 \pi r^{2} \exp (a t) \Omega[r \exp (a t / 2)],
$$

is the circulation around a circle of radius $r$. Note that $\omega$ has no dependence on the axial coordinate $z$. We will subsequently refer to the nonaxisymmetric Fourier components (2) with $n \neq 0$ as the spiral and the axisymmetric component (3) with $n=0$ as the core. An alternative derivation of (1)-(3) to that given by Lundgren ${ }^{5}$ is outlined in Appendix A.

An important approximation is the repression of the explicit $\tau$ dependence of $\Omega(\rho, \tau)$ in (1)-(6). This is justified by Lundgren ${ }^{5}$ (Appendix $A$ ), who shows that for the inviscid spiral evolution in the stretched space and time variables

$$
\Omega(\rho, \tau)=\Omega(\rho, 0)+O\left(\tau^{-2}\right) .
$$

Hence $\Omega$ is constant to order $\tau^{-2}$ and so in (1)-(6) $\Omega(\rho, \tau)$ may be replaced by $\Omega(\rho)=\Omega(\rho, 0)$ with error $O\left(\tau^{-2}\right)$. A similar approximation is made for the $\tau$ dependence $f(\rho, \tau)$ where it appears in (2) and (5), and for $g(\rho, \tau)$ in $(5)$. Where these functions appear in the axisymmetric component of the vorticity distribution (3), the full $\tau$ dependence produced by viscous effects will be retained.

Some remarks help clarify the meaning of the (1)-(4). When $v=0$, the vorticity distribution (1) - (3) may be written in the form

$$
\omega(r, \theta, t)=2 \pi e^{a t} f(\rho) \delta[\theta-\Omega(\rho) \tau]+e^{a t} g(\rho),
$$

where $\delta(\cdots)$ denotes the Dirac delta function. Equation (8) describes a vortex sheet in the process of being rolled up with angular velocity $\Omega$ by the total circulation $\Gamma(r, t)$ which lies inside a point on the spiral and which is the sum of contributions from the spiral and from the background vorticity. This requires that $\Omega$ be monotone decreasing, i.e., $\Lambda \leqslant 0$. The vortex sheet evolves dynamically according to the equation $\theta=\Omega(\rho) \tau$. At the same time the sheet is being compressed in the radial direction by the inward radial component of the strain field. As a result of simultaneous roll-up and radial compression the radial distance between successive turns of radius $r$ at time $t$ is

$$
\delta r=\frac{2 \pi \exp (-a t / 2)}{\Lambda[r \exp (a t / 2)] \tau(t)} .
$$

The viscous term in (2) describes the diffusion of vorticity between individual turns.
The solution (1)-(4) is asymptotically valid for large time. When $t \rightarrow 0$ the vorticity is concentrated on the vortex sheet but the approximate solution has no meaning as a global vorticity distribution because the formation mechanism is not described explicitly. Some memory of formation is retained, however, in $f$. For example vortex sheet roll-up into a Kaden-like spiral would produce $f \propto \rho^{\alpha}$ where $\alpha \leqslant 0$ is some exponent that depends on the initial vortex-sheet structure (see Moore and $\operatorname{Saffman}^{20}$ and Pullin $\left.{ }^{21}\right)$. We will view the spiral as being created at time $t_{1} \approx 1 / a$ [we will later take $\tau_{1}=\phi / a$ (where $\phi$ is a parameter) when the thickness of each turn is of order the Burgers vortex thickness $\Delta=(v / a)^{1 / 2}$ ]. Owing to the presence of the real exponent in the exponential of (2) the Fourier modes representing the spiral have a finite lifetime. Thus an individual turn becomes smoothed by viscosity when the radial distance to the nearest other turn is of $O(\Delta)$. At time $t$ the spiral vortex as a whole consists of an inner viscous subcore of radius $r_{\mathrm{I}}=O(\Delta)$ where vortex stretching and viscous diffusion of vorticity are in local balance, an intermediate region of radius $r_{\mathrm{II}}(t)$ [obtained as a solution of (9) when the left-hand side is replaced by $\Delta]$, where viscous diffusion has merged individual turns into a smoothed axisymmetric rotational core with vorticity ex$\mathrm{p}(a t) f(\rho)$, and an outer region consisting of individual spiral turns and of radius $r_{\mathrm{III}}(t)=R \exp (-a t / 2)$ where $R$ is a measure of the initial radius of the whole vortex. The spiral exists until $r_{\mathrm{II}}=r_{\mathrm{III}}$. An analysis of this structure for the generalized Kaden spiral is given by Moore and Saffman. ${ }^{20}$

\section{B. The energy spectrum}

The energy spectrum is calculated using known relationships in the theory of homogeneous turbulence bctween $E(k, t)$, the instantaneous average turbulent kinetic energy per unit mass at wave number $k=|\mathbf{k}|$, where $\mathbf{k}$ is the vector wave number, and the power spectrum of the vorticity field. Assuming further that two-point one-time vorticity correlations are finite only within individual vortices, that the vortex centerline curvature is large compared with $1 / R$, and that $\omega$ is independent of the axial coordinate, the time-averaged spectrum for a general $\omega(r, \theta, t)$ within individual vortices given by Lundgren ${ }^{5}$ may be expressed in the form

$$
\begin{aligned}
& E(k)=E_{0}(k)+E_{s}(k), \\
& E_{0}(k)=\frac{\pi N}{k} \int_{0}^{\tau_{2}} \hat{I}_{0}^{2}(k, \tau) d \tau, \\
& E_{s}(k)=\frac{2 \pi N}{k} \int_{\tau_{1}}^{\infty} \sum_{n=1}^{\infty}\left|\hat{I}_{n}(k, \tau)\right|^{2} d \tau, \\
& \hat{I}_{n}(k, \tau)=\int_{0}^{\infty} J_{n}(k r) \omega_{n}(r, t) r d r .
\end{aligned}
$$

In (10)-(13) $N$, which has the dimensions length ${ }^{-2}$ time ${ }^{-1}$, is the rate of creation of vortex length per unit volume per unit time, $E_{0}(k)$ is the contribution to the spectrum from the axisymmetric part of the vorticity [i.e., 
from (3)], and $E_{s}(k)$ is the contribution from the spiral. In Appendix B we give an alternative derivation of (10)-(13) to that developed by Lundgren. ${ }^{5}$ In (11) the lower limit of integration has been set to zero since the integral is expected to converge at $\tau=0$ while in (12) the upper limit is set to $\infty$ since the spiral has a finite life and the integral again converges. The lower limit in (12) is $\tau_{1}=\phi / a$. The upper limit of integration $\tau_{2}$ in (11) will be discussed subsequently. In obtaining (10)-(13) an ergodic hypothesis was invoked by assuming that all vortices undergo an identical evolution process. If the number per unit time per unit volume at some particular stage in the evolution is stationary, this then allows averages over the spatial ensemble of vortices with different ages to be replaced by a time integration over a single vortex history. If several classes or a distribution of vortices of differing type exist then (10) requires an additional sum over these classes or distributions. Note that we have and will continue for convenience to express time integrals in terms of the stretched time variable $\tau$.

Lundgren then uses (1)-(3) in (10)-(13), using stationary phase to evaluate the integrals (13), to obtain a specific form of $E_{s}(k)$ for the evolving spiral vortex. This may be expressed as

$$
\begin{aligned}
& E_{s}(k)=A k^{-5 / 3} \exp \left(-\frac{2 v k^{2}}{3 a}\right) \\
& A=\frac{4 \pi}{3} N a^{1 / 3} \int_{0}^{\infty} \frac{|f(\rho)|^{2} \rho d \rho}{|\Lambda(\rho)|^{4 / 3}} \sum_{n=1}^{\infty} n^{-4 / 3} .
\end{aligned}
$$

In the passage from (12) to (15) $\tau_{1}$ in (12) has been set to zero again on the grounds that within the bounds of the approximations made the resulting integral over the argument of the $f$ converges at $\tau=0$. We remark that the $-5 / 3$ exponent in (14) emerges only after the time integration in (11), and so the ergodic hypothesis is of crucial importance. The instantaneous energy spectrum of a single vortex at some time in its evolution is not generally of the $k^{-5 / 3}$ form.

Equations (1)-(15) can readily be extended to a system of $M$ interleaved spiral layers, each with a different phase. A further extension in which $f(\rho)$ in (2) is replaced by functions $f_{n}(\rho)$ displaying dependence on $n$ preserves the form of (14) but modifies (15) by shifting the integral inside the sum. In this case the structure may not resemble a rolled-up shear layer of the type described in Sec. II $\mathrm{A}$ but is of a more general spiral form. The essential property of the vorticity distribution (1)-(4) required for a spectrum like (14) is contained in the phase-amplitude relations of the Fourier coefficients.

If, following Townsend, ${ }^{1}$ we substitute into $(10)$ and (11) the steady axisymmetric Burgers vortex solution

$$
\omega_{0}(r)=\frac{a \Gamma_{0}}{4 \pi v} \exp \left(-\frac{r^{2} a}{4 v}\right),
$$

[see Burgers; ${ }^{22}$ Rott $^{23}$ gives a discussion of (16)] we obtain the spectrum

$$
E_{0}(k)=\frac{N \tau_{2} \Gamma_{0}^{2}}{4 \pi k} \exp \left(-\frac{2 k^{2} v}{a}\right),
$$

and of course there is no nonaxisymmetric contribution. The dissipation for this spectrum is (see Sec. IV A)

$$
\epsilon=3 a^{2} v+\frac{a N \tau_{2} \Gamma_{0}^{2}}{8 \pi},
$$

where the first term on the right-hand side is the dissipation per unit mass by the background strain field. The second term is the dissipation of the Burgers vortex. This is unbounded when $\tau_{2} \rightarrow \infty$ owing to the unbounded $z$ extension of vortex lines by the strain. If again following Townsend ${ }^{1}$ it is assumed that $a^{2} v / \epsilon=1 / C^{2}$ where $C$ is a dimensionless constant then when $a \tau_{2}$ is large the background dissipation is small and (17) and (18) may be combined to give

$$
\frac{E}{\left(\epsilon v^{5}\right)^{1 / 4}}=\frac{2 C}{k \eta} \exp \left(-2 C k^{2} \eta^{2}\right) \text {. }
$$

\section{SPIRAL MODEL WITH ZERO TOTAL CIRCULATION}

\section{A. A specific spiral model}

Quantitative calculation with the Lundgren model requires choices for $f$ and $g$. We assume a model where these functions are of the form

$$
\begin{aligned}
& f(\rho)=\frac{\Gamma_{0}}{R^{2}} \widetilde{f}(\xi), \quad g(\rho)=\frac{\Gamma_{0}}{R^{2}} \widetilde{g}(\xi), \\
& \Omega(\rho)=\frac{\Gamma_{0}}{R^{2}} \widetilde{\Omega}(\xi), \quad \Lambda(\rho)=\frac{\Gamma_{0}}{R^{3}} \widetilde{\Lambda}(\xi),
\end{aligned}
$$

where $\xi=\rho / R, \Gamma_{0}$ is a circulation scale, and $R$ is a measure of the initial lateral scale of the vortex structure. A specific choice for $\widetilde{f}(\xi), \widetilde{g}(\xi)$ is made as

$$
\begin{aligned}
& \tilde{f}(\xi)=\frac{1}{\pi \Gamma[1+\alpha / 2]} \xi^{\alpha} e^{-\xi^{2},} \\
& \tilde{g}(\xi)=-\frac{1}{\pi} \xi^{2} e^{-\xi^{2}},
\end{aligned}
$$

where $\Gamma[\cdots]$ is the gamma function and we use square brackets to denote an argument. While other choices for $f$ and $g$ were considered, Eqs. (22) and (23) give a simple model motivated as follows: we envision the vortex-sheetlike roll-up of sheetlike structures to give a power law $f$ with exponent $\alpha$. We invoke ideas of Onsager ${ }^{24}$ and suppose this nearly one-signed vorticity will then attract debris with vorticity of opposite sign which we model as a background halo represented by $g(\xi)$ superposed on the spiral vortex. We require

$$
2 \pi \int_{0}^{\infty} f(\xi) \xi d \xi-2 \pi \int_{0}^{\infty} g(\xi) \xi d \xi=1
$$

i.e., it is assumed that the spiral attracts just sufficient vorticity of opposite sign so that the composite vortex has zero total circulation $[\Gamma(\infty)=0]$. The background vorticity $g$ is 


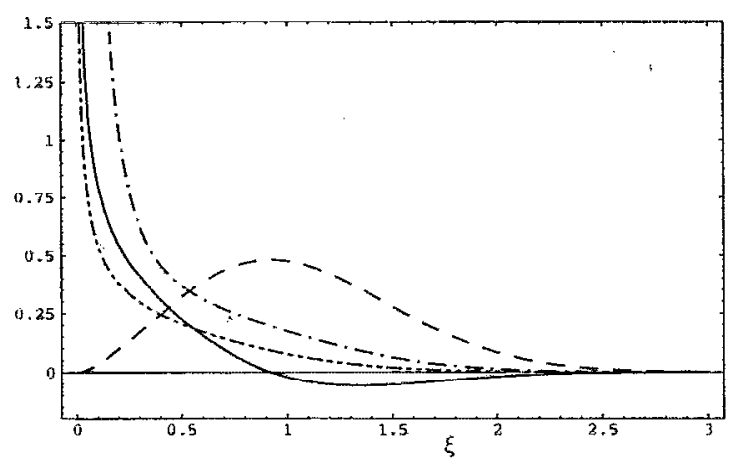

FIG. 1. Dimensionless spiral functions versus $\xi=\rho / R$. $-: \tilde{f}+\tilde{g}$

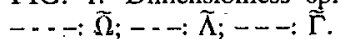

not concentrated near the origin but is confined to the outer part of the composite structure. A radial cut through the contracting vortex would reveal an oscillating vorticity distribution not unlike the cross section of a vortex tube obtained by Vincent and Meneguzzi ${ }^{14}$ in $240^{3}$ isotropic turbulence simulations at a Taylor-Reynolds number $R_{\lambda} \approx 150$ and shown in Fig. 15 of their paper. Putting the real exponent of the exponential in (2) equal to unity then gives an estimate of the spiral lifetime $\tau_{\text {spiral }}$ as

$$
(a \tau)_{\text {spiral }} \sim\left(\frac{R^{2} a}{4 v}\right)\left(\frac{\Gamma_{0}}{v}\right)^{-2 / 3} .
$$

While the spirals themselves automatically have a finite lifetime, (24) ensures that the core also dies at large $t$ by a process of strain-enhanced self-cancellation. We view this as a model of a finite life for the composite vortex structure without which the core will collapse into a steady and continuously stretching and dissipating equilibrium Burgers vortex. In such a structure $E_{0}$ would be dominant at large $t$. The spectrum would then be asymptotic to (17) and there would be no inertial range. We note that Douady et $a l^{15}$ observe intense short-lived vortex tubes in laboratory experiments.

A referee has suggested that vortex reconnection may provide an alternative mechanism for limiting the lifetime of vortex cores when $\Gamma(\infty) \neq 0$. We do not explore this possibility as there is no satisfactory quantitative theory of reconnection which can estimate $\tau_{2}$. Presently we prefer employing viscosity in a mathematically consistent way rather than introducing an ad hoc cutoff $\tau_{2}$, which is not easily related to the structure of the vortices, to describe the effect of reconnection or similar mechanisms.

We can obtain $\widetilde{\Omega}(\xi)$ from (5) and (22) and (23) which gives

$$
\widetilde{\Omega}(\xi)=\frac{1}{2 \pi \xi^{2}}\left(e^{-\xi^{2}}\left(1+\xi^{2}\right)-1+\frac{\gamma\left[1+\alpha / 2, \xi^{2}\right]}{\Gamma[1+\alpha / 2]}\right),
$$

where $\gamma[$,$] is the incomplete gamma function. Figure 1$ shows plots of the total $\theta$-averaged vorticity $\widetilde{f}(\xi)+\widetilde{g}(\xi)$, together with $\widetilde{\Omega}(\xi), \widetilde{\Lambda}(\xi)$, and the cumulative dimensionless circulation $\widetilde{\Gamma}(\xi)=\Gamma(\rho / R) / \Gamma_{0}$ for the choice $\alpha$ $=-0.5$. The negative circulation gradient in the outer part
TABLE I. Values of $B_{0}, D_{0}$, and $\left(\Gamma_{0} / v\right)_{c}$ for three values of $\alpha$.

\begin{tabular}{cccc}
\hline$\alpha$ & $D_{0}$ & \multicolumn{1}{c}{$B_{0}$} & $\left(\Gamma_{\alpha} / v\right)_{c}$ \\
\hline-0.25 & 12.19 & 0.00657 & 363.1 \\
-0.5 & 7.029 & 0.00848 & 198.4 \\
-1.0 & 2.214 & 0.0135 & 58.8 \\
\hline
\end{tabular}

of the structure suggests a possible dynamical mechanism for finite vortex lifetime via a Rayleigh-type instability which we have not presently analyzed.

\section{B. Spectrum of the spiral}

The spectrum of the spiral may now be calculated explicitly. Using (20)-(23) and (26) in (15) gives $E_{s}(k)$ which we write in the form

$$
\begin{aligned}
& E_{s}(k)=D_{0}(\alpha) N R^{2} \Gamma_{0}^{2 / 3} a^{-1 / 2} v^{5 / 6} K^{-5 / 3} \exp \left(-K^{2} / 6\right), \\
& D_{0}(\alpha)=\frac{2^{11 / 3} \pi}{3} \int_{0}^{\infty} \frac{\widetilde{f}^{2}(\xi)}{|\widetilde{\Lambda}(\xi)|^{4 / 3}} \xi d \xi \sum_{1}^{\infty} n^{-4 / 3}
\end{aligned}
$$

where $K=2 k(v / a)^{1 / 2}$ is the dimensionless wave number and $\widetilde{\Lambda}(\xi)=d \widetilde{\Omega}(\xi) / d \xi$. In (27) and (28) $D_{0}$ is a pure number that can be calculated using (21)-(23) and (26). We note that both the integral and sum in (28) are convergent. Table 1 shows values of $D_{0}$ for $\alpha=-0.25,-0.5$, and -1.0 . These and other numerical and symbolic calculations reported presently were done independently using the symbolic manipulators MATHEMATICA 2.05 and MAPLE version 5 .

\section{Spectrum of the core}

To determine the core spectrum $E_{0}(k)$ we first solve the axisymmetric time-dependent vorticity equation in the presence of the strain field $\left(u_{r} u_{\theta}, u_{z}\right)=(-a r / 2,0, a z)$

$$
\frac{\partial \omega_{0}(r)}{\partial t}-\frac{a r}{2} \frac{\partial \omega_{0}(r)}{\partial r}=a \omega+\frac{v}{r} \frac{\partial}{\partial r}\left(r \frac{\partial \omega_{0}}{\partial r}\right),
$$

with initial conditions given by the $\theta$-averaged vorticity field at $t=0, \omega_{0}(r, 0)=f(r)+g(r)$. The solution may be obtained via Green's functions and is given by

$$
\begin{aligned}
\omega_{0}(r, t)= & \frac{e^{a t}}{2 v \tau} \exp \left(\frac{-r^{2} e^{a t}}{4 v \tau}\right) \int_{0}^{\infty}[f(u)+g(u)] \\
& \times \exp \left(-\frac{u^{2}}{4 v \tau}\right) I_{0}\left(\frac{r e^{a t / 2} u}{2 v \tau}\right) u d u,
\end{aligned}
$$

where $I_{0}$ is the modified Bessel function of order zero and $\tau(t)$ is given by the second of (4). There is no inconsistency with our previous assumption (7) as the evolution of $\omega_{0}$ is independent of $\Omega$. On substitution of (30) into (13), the $r$ integration can be performed to give 


$$
\begin{aligned}
\hat{I}_{0}(k, \tau)= & \exp \left(-\frac{v \tau k^{2}}{1+a \tau}\right) \int_{0}^{\infty}[f(u)+g(u)] J_{0} \\
& \times\left(\frac{k u}{\sqrt{1+a \tau}}\right) u d u .
\end{aligned}
$$

Using (20) and (22) and (23) the $u$ integration can be performed, and on putting $v=a \tau$ we find

$$
\begin{gathered}
\int_{0}^{\infty}[f(u)+g(u)] J_{0}\left(\frac{k u}{\sqrt{1+a \tau}}\right) u d u=\Gamma_{0} H(k R, \alpha ; v), \\
H(k R, \alpha ; v)=\frac{1}{2 \pi} \exp \left(-\frac{k^{2} R^{2}}{4(1+v)}\right)\left[\frac{k^{2} R^{2}}{4(1+v)}\right. \\
\left.-1+M\left(-\frac{\alpha}{2}, 1, \frac{k^{2} R^{2}}{4(1+v)}\right)\right],
\end{gathered}
$$

where $M(,$,$) is the confluent hypergeometric function.$ We now make the approximation $\tau /(1+a \tau) \approx 1 / a$ in the leading exponential of (31) and substitute (31) and (32) into (11). After some straightforward algebra and some rearrangement of terms $E_{0}(k)$ may be expressed as

$$
E_{0}(k)=B_{0}(\alpha ; k R) N R^{2} \Gamma_{0}^{2} a^{-1 / 2} v^{-1 / 2} K \exp \left(-K^{2} / 2\right),
$$

where

$$
B_{0}(\alpha ; k R)=\frac{\pi}{2} \int_{0}^{\infty} \frac{1}{k^{2} R^{2}} H^{2}(k R, \alpha ; v) d v .
$$

Note that $\tau_{2}$ in (11) may now be set to $\infty$ since (24) ensures that the core self-cancels for large time by strainenhanced viscous diffusion. When $\alpha$ is fixed and $k R \gg 1$ numerical calculation shows that the pure number $B_{0}$ given by (35) becomes independent of $k R$ and so, for large $k R, E_{0}$ is of the form $k \exp \left(-2 k^{2} v / a\right)$.

It will be later shown that for the present model $(v / a)^{1 / 2}$ is proportional to $\eta$; thus $K$ is proportional to $k \eta$. Dividing (34) by (27) and evaluating the result at $K=1$ then gives the ratio $E_{0} / E_{s}$ at the point where, from (34), $E_{0}$ is maximum

$$
\frac{E_{0}(K=1)}{E_{s}(K=1)}=\frac{B_{0}}{D_{0}} e^{-1 / 3}\left(\frac{\Gamma_{0}}{v}\right)^{4 / 3} .
$$

If this ratio were to exceed unity then the total spectrum $E_{0}(K)+E_{s}(K)$ would exhibit a large bump near the Kolmogorov wave number which is not observed either experimentally or in numerical simulation. Hence for the model we restrict $\Gamma_{0} / v$ to values satisfying $\Gamma_{0} / v<\left(\Gamma_{0} / v\right)_{c}$ where $\left(\Gamma_{0} / v\right)_{c}$ is obtained from (36) by fixing the left-hand side to unity. Table I shows values of $B_{0}, D_{0}$, and $\left(\Gamma_{0} / v\right)_{c}$ for our preferred value of $\alpha=-0.5$ and also for $\alpha=-0.25$, -1.0 . We note that for $\alpha=-0.25$ and -0.5 , the calculated $\left(\Gamma_{0} / v\right)_{c}$ are of the same order as the $\Gamma_{0} / v$ found by Jiménez ${ }^{7}$ to be characteristic of small-scale vortices in a variety of turbulent flows.

At this stage the model contains five dimensional parameters, namely $N, R, a, v$, and $\Gamma_{0}$. We comment that the functional dependence on these parameters displayed in
(27) and (34) is generic to the Lundgren model provided $\Gamma(\infty)=0$, but it is clear from (28) and (35) that the numerical values of $D_{0}$ and $B_{0}$ depend on the particular choice of $f$. If $\Gamma(\infty) \neq 0$ the form (27) remains but the core spectrum for large $k R$ is of the form $k^{-1} \exp \left(-2 k^{2} v / a\right)$.

\section{KOLMOGOROV PREFACTOR AND SKEWNESS}

\section{A. The dissipation}

From the five physical parameters may be formed the three dimensionless groups

$$
\frac{N R^{2}}{a}, \frac{\Gamma_{0}}{v}, \frac{R^{2} a}{4 v} .
$$

We discuss these groups by first calculating the dissipation

$$
\epsilon=3 v a^{2}+2 v \int_{0}^{\infty} k^{2} E_{0}(k) d k+2 v \int_{0}^{\infty} k^{2} E_{s}(k) d k .
$$

In (37) the first term is dissipation per unit mass in the background. This may be viewed as originating from vorticity effectively placed at infinity (the collection of vortex tubes or the vorticity in the larger scales) providing the local strain. When $K$ in (27) is expressed in terms of $k$, the Kolmogorov prefactor $\mathscr{K}_{0}$ may be written

$$
\mathscr{K}_{0} \epsilon^{2 / 3}=2^{-5 / 3} D_{0}(\alpha) N R^{2} \Gamma_{0}^{2 / 3} a^{1 / 3} .
$$

\section{B. The skewness $\boldsymbol{S}_{3}$}

An exact equation for decaying isotropic turbulence with time variable $T$ is

$$
\frac{1}{35} \frac{d}{d T}\left(\overline{\overline{\omega^{2}}}\right)=\overline{\overline{\left(\frac{\partial u}{\partial x}\right)^{3}}}+\frac{4 v}{35} \int_{0}^{\infty} k^{4} E(k, T) d k .
$$

Equation (39) can be obtained from the von KármánHowarth equation (see Batchelor ${ }^{25}$ ) in the limit of zero spatial separation between two points in the fluid. In (39) $\partial u / \partial x$ is a longitudinal velocity derivative and " an ensemble average. In isotropic turbulence theory, scaling arguments (e.g., Batchelor ${ }^{25}$ ) are usually invoked to show that the term on the left-hand side of (39) is small so that the terms on the right-hand side which refer, respectively, to the stretching of vortex lines by the local strain field and the dissipation of the mean square vorticity by viscosity are in local balance. We may use (39) presently by first identifying $T$ with $t$ and integrating both sides in $\left(t_{1}, \infty\right)$. Next (1)-(4) may be used to show that the lefthand side of the resulting equation evaluated at $t=t_{1}$ is small compared to the remaining terms. Finally the contribution of the background strain field to the cubic longitudinal derivative is added to give

$$
\overline{\overline{\left(\frac{\partial u}{\partial x}\right)^{3}}}=\frac{2 a^{3}}{35}-\frac{4 v}{35} \int_{0}^{\infty} k^{4}\left[E_{0}(k)+E_{s}(k)\right] d k \text {. }
$$

The leading term on the right-hand side of $(40)$ has been evaluated from the strain field with principal rates of strain 
TABLE II. Calculated values of $a^{2} v / \epsilon, \mathscr{K}_{0}$, and $S_{3}$ for fixed $\alpha, \Gamma_{0} / v$, and $N R^{2} / a$.

\begin{tabular}{ccclcc}
\hline \hline & $\Gamma_{0}$ & $\frac{N R^{2}}{a}$ & \multicolumn{1}{c}{$\frac{a^{2} v}{\epsilon}$} & $\mathscr{K}_{0}$ & \multicolumn{1}{c}{$S_{3}$} \\
\hline-0.25 & 100 & 0.2 & 0.0257 & 1.44 & -0.477 \\
-0.25 & 100 & 0.4 & 0.0134 & 1.86 & -0.363 \\
-0.25 & 100 & 0.6 & 0.00902 & 2.15 & -0.304 \\
-0.25 & 200 & 0.2 & 0.0132 & 1.46 & -0.361 \\
-0.25 & 200 & 0.4 & 0.00672 & 1.87 & -0.265 \\
-0.25 & 200 & 0.6 & 0.00451 & 2.15 & -0.219 \\
-0.5 & 100 & 0.4 & 0.0186 & 1.34 & -0.419 \\
-0.5 & 100 & 0.6 & 0.0126 & 1.55 & -0.354 \\
-0.5 & 100 & 0.8 & 0.00956 & 1.78 & -0.310 \\
-0.5 & 150 & 0.4 & 0.0117 & 1.29 & -0.342 \\
-0.5 & 150 & 0.6 & 0.00789 & 1.46 & -0.286 \\
-0.5 & 150 & 0.8 & 0.00600 & 1.64 & -0.250 \\
-1.0 & 30 & 3.0 & 0.0175 & 1.37 & -0.409 \\
-1.0 & 30 & 4.0 & 0.0133 & 1.51 & -0.363 \\
-1.0 & 30 & 5.0 & 0.0107 & 1.64 & -0.329 \\
\hline \hline
\end{tabular}

$(-a / 2,-a / 2, a)$ using the standard Betchov ${ }^{26} /$ Batchelor-Townsend $^{27}$ formula (see also Sec. V A).

The skewness $S_{3}$ is

$$
S_{3}=\frac{\overline{\overline{(\partial u / \partial x)^{3}}}}{\overline{\overline{\left((\partial u / \partial x)^{2}\right)^{3 / 2}}}}
$$

where

$$
\overline{\left(\frac{\partial u}{\partial x}\right)^{2}}=\frac{\epsilon}{15 v} .
$$

\section{Setting the parameters}

Use of (27) and (34) in (37) and (38) and (40)-(42) and the rewriting of (38) then gives three equations which, after some rearranging may be written in the form

$$
\begin{aligned}
& 3\left(\frac{a^{2} v}{\epsilon}\right)+\mathscr{K}_{0}\left\{\frac{2^{2 / 3} B_{0}}{D_{0}}\left(\frac{\Gamma_{0}}{v}\right)^{4 / 3}+\frac{3}{2} \Gamma\left[\frac{2}{3}\right]\right\}\left(\frac{a^{2} v}{\epsilon}\right)^{1 / 3}=1 \\
& \mathscr{K}_{0}=2^{-5 / 3} D_{0}\left(\frac{N R^{2}}{a}\right)\left(\frac{a^{2} v}{\epsilon}\right)^{2 / 3}\left(\frac{\Gamma_{0}}{v}\right)^{2 / 3}, \\
& S_{3}=\frac{6 \sqrt{15}}{7}\left(\frac{a^{2} v}{\epsilon}\right)^{3 / 2}-\frac{12 \sqrt{15}}{7} \mathscr{K}_{0}\left\{\frac{B_{0}}{2^{1 / 3} D_{0}}\left(\frac{\Gamma_{0}}{v}\right)^{4 / 3}\right. \\
& \left.+\frac{1}{2}\left(\frac{3}{2}\right)^{5 / 3} \Gamma\left[\frac{5}{3}\right]\right\}\left(\frac{a^{2} v}{\epsilon}\right)^{5 / 6} .
\end{aligned}
$$

and we note that (43) and (45) depend on the exponential tail behavior in (27) and (34).

When $B_{0}$ and $D_{0}$ are fixed (i.e., $\alpha$ fixed) (43)-(45) are three equations for the five quantities $\mathscr{K}_{0}, S_{3}, N R^{2} / a$, $\Gamma_{0} / \nu$, and a new dimensionless group $a^{2} v / \epsilon$. We may therefore fix values of two of these and calculate the remaining three. Table II shows results obtained by setting values of $\Gamma_{0} / v$ and $N R^{2} / a$, and calculating $\mathscr{K}_{0}, S_{3}$, and $a^{2} v / \epsilon$. Values of $B_{0}$ and $D_{0}$ used are from Table I. We will

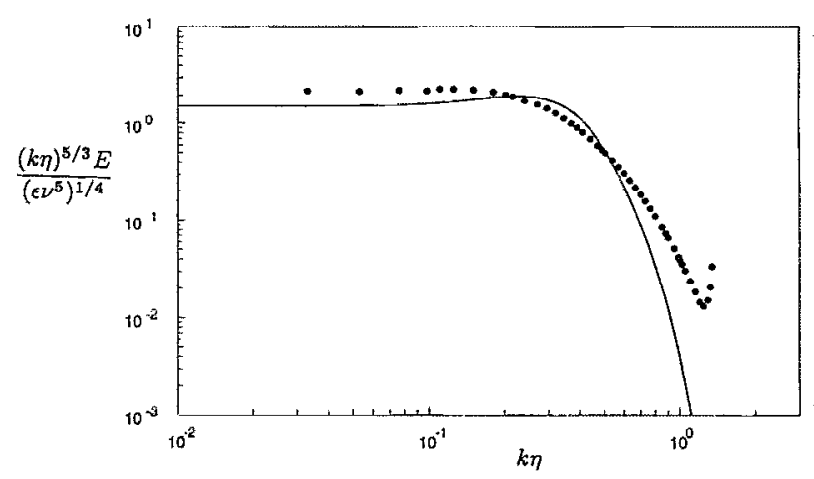

FIG. 2. Energy spectrum of the form $k^{5 / 3} E$ vs $k \eta$. $=-0.5, \Gamma_{0} / v=100$, and $N R^{2} / a=0.6 ;-128^{3}$ numerical simulation, Kerr, ${ }^{10} R_{\lambda}=82.9$.

select and use subsequently $\alpha=-0.5, \Gamma_{0} / v=100$, and $N R^{2} / a=0.6$, for which the calculated $\mathscr{K}_{0}=1.52$ and $S_{3}=-0.35$ are within the range of experiment (see Yaglom $^{28}$ for experimental $\mathscr{K}_{0}$ and Van Atta and Antonia ${ }^{19}$ for a compilation of experimental results for $S_{3}$ ). We note that our value of $\Gamma_{0} / v$ is of the same order as but somewhat smaller than the estimates of Ashurst ${ }^{6}$ and Jiménez, ${ }^{7}$ and for this reason we will also perform some calculations using $\alpha=-0.25, \quad \Gamma_{0} / v=200, \quad$ and $N R^{2} / a=0.2$. For both these choices the calculated $S_{3}$ is smaller in magnitude than $S_{3} \approx-0.5$ found in $128^{3}$ homogeneous turbulence simulations of $\operatorname{Kerr}^{9}$ and the $240^{3}$ simulations of Vincent and Meneguzzi. ${ }^{14}$ Note that $R^{2} a / 4 v$ does not appear in (43)-(45). This parameter has the form of a (vortex) Reynolds number which will be later (see Sec. VI D) related to the Taylor microscale Reynolds number $R_{\lambda}$. Hence within the present model there is no dependence of the five parameters discussed above on $R_{\lambda}$. This agrees with results of the numerical simulations for $\mathscr{K}_{0}$ and $S_{3}$. The calculated $a^{2} v / \epsilon=0.0126$ (which may be compared with the Townsend ${ }^{1}$ assumed value $\left.a^{2} \nu / \epsilon=1 / 15\right)$ then gives the Kolmogorov length as $\eta=(v / a)^{1 / 2}\left(a^{2} v / \epsilon\right)^{1 / 4} \approx 0.33(v / a)^{1 / 2}$.

The spiral and the core contributions to the energy spectra may be expressed in a standard form in terms of $k \eta$ as

$$
\begin{aligned}
& \frac{E_{0}(k \eta)}{\left(\epsilon v^{5}\right)^{1 / 4}}= \frac{2^{8 / 3} B_{0}(\alpha)}{D_{0}(\alpha)} \mathscr{K}_{0}\left(\frac{\Gamma_{0}}{v}\right)^{4 / 3}\left(\frac{a^{2} v}{\epsilon}\right)^{2 / 3}(k \eta) \\
& \times \exp \left(-17.8 k^{2} \eta^{2}\right), \\
& \frac{E_{s}(k \eta)}{\left(\epsilon v^{5}\right)^{1 / 4}=}=\mathscr{K}_{0}(k \eta)^{-5 / 3} \exp \left(-5.94 k^{2} \eta^{2}\right) .
\end{aligned}
$$

With the parameter values discussed above, (46) and (47) may be summed to give the overall spectrum in the form

$$
\begin{aligned}
\frac{E}{\left(\epsilon v^{5}\right)^{1 / 4}}= & \mathscr{K}_{0}\left[67.8(k \eta) \exp \left(-17.8 k^{2} \eta^{2}\right)\right. \\
& \left.+(k \eta)^{-5 / 3} \exp \left(-5.94 k^{2} \eta^{2}\right)\right],
\end{aligned}
$$

where $\mathscr{K}{ }_{0}=1.52$. Figure 2 shows (48) plotted as 


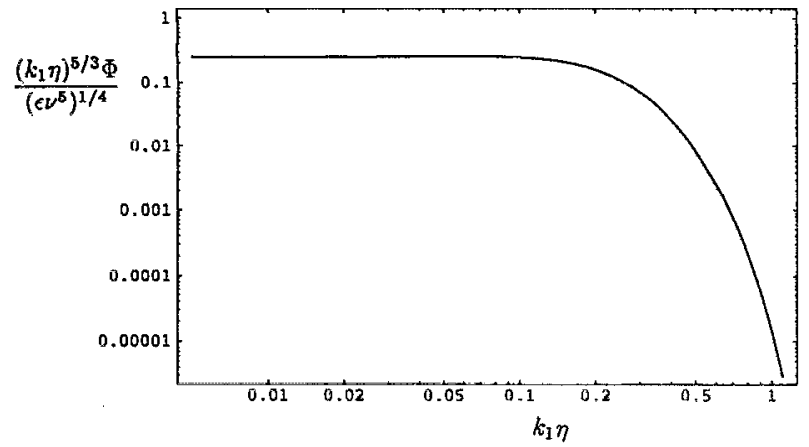

FIG. 3. Longitudinal one-dimensional spectrum $k^{5 / 3} \Phi$ vs $k \eta, \alpha=-0.5$, $\Gamma_{0} / \nu=100$, and $N R^{2} / a=0.6$.

$(k \eta)^{5 / 3} E /\left(\epsilon v^{5}\right)^{1 / 4}$ compared with the spectrum calculated by $\mathrm{Kerr}^{9,10}$ in $128^{3}$ numerical simulations of homogeneous turbulence at $R_{\lambda}=82.9$. Kerr comments on the presence of a small bump in the spectrum near the transition to the dissipation range. While smaller than that found presently (owing to the effect of the axisymmetric component of the spectrum), this can nevertheless be seen in the data. We remark that neither (48) nor (17) contain an $\exp (-B k \eta)$ range ( $B$ is a dimensionless constant) as suggested by the $128^{3}$ isotropic simulations of Kerr. ${ }^{10}$ However, (14) agrees with the preferred form suggested by Smith and Reynolds. ${ }^{29}$

Figure 3 shows the longitudinal one-dimensional spectrum calculated from (48) using the standard result (see Batchelor, ${ }^{25}$ p. 50)

$$
\Phi\left(k_{1}\right)=\frac{1}{2} \int_{k_{1}}^{\infty}\left(1-\frac{k_{1}^{2}}{y^{2}}\right) \frac{E(y)}{y_{1}} d y .
$$

In the dissipation range this curve falls below the compiled experimental data of Chapman. ${ }^{30}$ It is notable that no bump is apparent in the $\Phi\left(k_{1}\right)$ spectrum. Finally we comment that ratio of the contributions to the dissipation from the background strain, the axisymmetric core, and the spiral are given by the ratios of the three terms on the lefthand side of (43). Fixing the background as unity, these ratios for our preferred values $\alpha=-0.5, \Gamma_{0} / v=100$, and $N R^{2} / a=0.6$ are 1:9:17. The dissipation within the viscous subcore of radius $(v / a)^{1 / 2}$ cannot be calculated explicitly but will be some fraction of the dissipation of the axisymmetric component of the spectrum. This subcore, rather than the axisymmetric core which itself is distributed over the whole vortex cross section, would in the present model correspond to the Burgers-like vortices proposed by Ashurst ${ }^{6}$ and Jiménez. ${ }^{7}$ Thus in the present model the Burgers subcore plays a minor if not passive role in the overall dynamics and contributes to but does not dominate the dissipation.

\section{THE VELOCITY-DERIVATIVE MOMENTS}

In order to calculate velocity-derivative and vorticity moments we need a means of estimating ensemble averages. Presently this is done by means of

$$
\overline{\overline{" \prime \prime}} \equiv \overline{\langle " "\rangle}
$$

where the right-hand side denotes two operations that are in general noncommutative. The first is an average over Euler angles at a point within a vortex structure denoted by 〈" "〉 followed by an integration over both the vortex cross section and the structure lifetime and denoted by "". The relation (50) is equivalent to an ergodic hypothesis combined with the assumption that the vortex structures filling space are oriented randomly in space. We describe the first operation below while leaving consideration of the second to Sec. VI A.

\section{A. Integration over Euler angles}

The one-point velocity derivative moments may be easily calculated for homogeneous turbulence. Consider a point in the fluid where the (right-handed) principal axes of strain rate are labeled $(1,2,3)$ with origin $O$ and corresponding principal rates of strain $\left(e_{1}, e_{2}, e_{3}\right)$ with $e_{1}+e_{2}+e_{3}=0$. Let $\left(\omega_{1}, \omega_{2}, \omega_{3}\right)$ be the components of the vorticity vector with respect to these axes. We will later place these axes within a spiral-vortex structure and specify $e_{1}, e_{2}$, and $e_{3}$ as functions of $r$ and $\theta$. Further let $\left(1^{\prime}, 2^{\prime}, 3^{\prime}\right)$ be laboratory axes, let $\phi$ be the (polar) angle between the $1-1^{\prime}$ axes and let $\chi$ be the (azimuthal) angle between the 1-O-1' plane and the $3^{\prime}$ axis. Then $\phi, \chi$ are two of three Euler angles describing the transformation matrix between the two axes systems. If the turbulence is isotropic then the $n$th moment of the longitudinal velocity gradient $\partial u / \partial x \equiv \partial u_{1^{\prime}} / \partial x_{1^{\prime}}$ is obtained by assuming random orientations of the $(1,2,3)$ system and integrating

$$
\begin{aligned}
\left\langle\left(\frac{\partial u}{\partial x}\right)^{n}\right\rangle= & \frac{1}{4 \pi} \int_{0}^{2 \pi} \int_{0}^{\pi}\left(e_{1} \sin ^{2} \phi \cos ^{2} \chi\right. \\
& \left.+e_{2} \sin ^{2} \phi \sin ^{2} \chi+e_{3} \cos ^{2} \phi\right)^{n} \\
& \times \sin \phi d \phi d \chi,
\end{aligned}
$$

and we note that integrals like (51) over Euler angles define $\langle "$ " $\rangle$. For the present model this corresponds to the random orientations of the vortex tubes. Performing the integrations for $n=1, \ldots, 12$ gives

$$
\begin{aligned}
& \left\langle\left(\frac{\partial u}{\partial x}\right)\right\rangle=0, \\
& \left\langle\left(\frac{\partial u}{\partial x}\right)^{2}\right\rangle=\frac{2\left(e_{1}^{2}+e_{2}^{2}+e_{3}^{2}\right)}{15}, \\
& \left\langle\left(\frac{\partial u}{\partial x}\right)^{3}\right\rangle=\frac{8\left(e_{1} e_{2} e_{3}\right)}{35}, \\
& \left\langle\left(\frac{\partial u}{\partial x}\right)^{4}\right\rangle=\frac{8\left(e_{1}^{4}+e_{2}^{4}+e_{3}^{4}\right)}{105}, \\
& \left\langle\left(\frac{\partial u}{\partial x}\right)^{5}\right\rangle=\frac{32 e_{1} e_{2} e_{3}\left(e_{1}^{2}+e_{2}^{2}+e_{3}^{2}\right)}{231},
\end{aligned}
$$




$$
\begin{aligned}
& \left\langle\left(\frac{\partial u}{\partial x}\right)^{6}\right\rangle=\frac{32\left[5\left(e_{1}^{6}+e_{2}^{6}+e_{3}^{6}\right)-3 e_{1}^{2} e_{2}^{2} e_{3}^{2}\right]}{3003} \\
& \left\langle\left(\frac{\partial u}{\partial x}\right)^{7}\right\rangle=\frac{32 e_{1} e_{2} e_{3}\left(e_{1}^{2}+e_{2}^{2}+e_{3}^{2}\right)^{2}}{429} \\
& \left\langle\left(\frac{\partial u}{\partial x}\right)^{8}\right\rangle=\frac{\left(e_{1}^{2}+e_{2}^{2}+e_{3}^{2}\right)\left[448\left(e_{1}^{6}+e_{2}^{6}+e_{3}^{6}\right)+1728 e_{1}^{2} e_{2}^{2} e_{3}^{2}\right]}{21879}
\end{aligned}
$$$$
\left\langle\left(\frac{\partial u}{\partial x}\right)^{9}\right\rangle=\frac{7168 e_{1} e_{2} e_{3}\left(e_{1}^{6}+e_{2}^{6}+e_{3}^{6}\right)-17408 e_{1}^{3} e_{2}^{3} e_{3}^{3}}{46189}
$$$$
\left\langle\left(\frac{\partial u}{\partial x}\right)^{10}\right\rangle
$$

$$
=\frac{1536\left(e_{1}^{10}+e_{2}^{10}+e_{3}^{10}\right)-1280 e_{1}^{2} e_{2}^{2} e_{3}^{2}\left(e_{1}^{4}+e_{2}^{4}+e_{3}^{4}\right)}{46189},
$$

$$
\begin{aligned}
& \left\langle\left(\frac{\partial u}{\partial x}\right)^{11}\right\rangle=\frac{46080 e_{1} e_{2} e_{3}\left(e_{1}^{8}+e_{2}^{8}+e_{3}^{8}\right)-143360 e_{1}^{3} e_{2}^{3} e_{3}^{3}\left(e_{1}^{2}+e_{2}^{2}+e_{3}^{2}\right)}{289731} \\
& \left\langle\left(\frac{\partial u}{\partial x}\right)^{12}\right\rangle=\frac{147456}{482885}\left(\frac{11}{120}\left(e_{1}^{12}+e_{2}^{12}+e_{3}^{12}\right)-\frac{1}{10} e_{1}^{2} e_{2}^{2} e_{3}^{2}\left(e_{1}^{6}+e_{2}^{6}+e_{3}^{6}\right)+\frac{89}{360} e_{1}^{4} e_{2}^{4} e_{3}^{4}\right) .
\end{aligned}
$$

Equations (52c) and (52d) agree with well-known results first obtained by Betchov ${ }^{26}$ and by Batchelor and Townsend. ${ }^{27}$ Putting $e_{1}=-a / 2, e_{2}=-a / 2, e_{3}=-a$ in (52c) gives the leading-order term on the right-hand side of (40).

The $2 p$ th moment of one component of vorticity $\omega_{x} \equiv \omega_{1}$, is

$$
\left\langle\omega_{x}^{2 p}\right\rangle=\frac{\left(\omega_{1}^{2}+\omega_{2}^{2}+\omega_{3}^{2}\right)^{p}}{2 p+1}, \quad p=1, \ldots,
$$

and we note that odd moments are necessarily zero.

The $n$th moment of the lateral velocity gradient $\partial v / \partial x \equiv \partial u_{2^{\prime}} / \partial x_{1^{\prime}}$ is

$$
\begin{aligned}
\left\langle\left(\frac{\partial v}{\partial x}\right)^{n}\right\rangle= & \frac{1}{8 \pi^{2}} \int_{0}^{2 \pi} \int_{0}^{2 \pi} \int_{0}^{\pi}\left(e_{1}(\sin \lambda \cos \phi \cos \chi+\cos \lambda \sin \chi)(\cos \lambda \cos \phi \cos \chi-\sin \lambda \sin \chi)\right. \\
& +e_{2}(-\cos \phi \sin \lambda \sin \chi+\cos \lambda \cos \chi)(-\cos \phi \cos \lambda \sin \chi-\sin \lambda \cos \chi)+e_{3} \sin \lambda \sin ^{2} \phi \cos \lambda \\
& \left.-\frac{1}{2} \omega_{1} \sin \phi \cos \chi+\frac{1}{2} \omega_{2} \sin \phi \sin \chi+\frac{1}{2} \omega_{3} \cos \phi\right)^{n} \sin \phi d \phi d \chi d \lambda,
\end{aligned}
$$

where here $\lambda$ is the third Euler angle. Symmetry arguments show that odd moments are zero. We have presently calculated the $n=2,4,6$ moments

$$
\begin{aligned}
\left\langle\left(\frac{\partial v}{\partial x}\right)^{2}\right\rangle= & \frac{e_{1}^{2}+e_{2}^{2}+e_{3}^{2}}{10}+\frac{\omega^{2}}{12} \\
\left\langle\left(\frac{\partial v}{\partial x}\right)^{4}\right\rangle= & \frac{3\left(e_{1}^{2}+e_{2}^{2}+e_{3}^{2}\right)^{2}}{140}+\frac{11 \omega^{2}\left(e_{1}^{2}+e_{2}^{2}+e_{3}^{2}\right)}{140}-\frac{3\left(\omega_{1}^{2} e_{1}^{2}+\omega_{2}^{2} e_{2}^{2}+\omega_{3}^{2} e_{3}^{2}\right)}{35}+\frac{\left(\omega^{2}\right)^{2}}{80} \\
\left\langle\left(\frac{\partial v}{\partial x}\right)^{6}\right\rangle= & \frac{1}{448}\left(\omega_{1}^{2}+\omega_{2}^{2}+\omega_{3}^{2}\right)^{3}+\frac{17}{616}\left(\omega_{1}^{2}+\omega_{2}^{2}+\omega_{3}^{2}\right)\left(e_{1}^{4}+e_{2}^{4}+e_{3}^{4}\right)-\frac{15}{308}\left(e_{1}^{2}+e_{2}^{2}+e_{3}^{2}\right)\left(\omega_{1}^{2} e_{2} e_{3}+\omega_{2}^{2} e_{3} e_{1}+\omega_{3}^{2} e_{1} e_{2}\right)+\frac{9}{154}\left(\omega_{1}^{2} e_{2}^{2} e_{3}^{2}\right. \\
& \left.+\omega_{2}^{2} e_{3}^{2} e_{1}^{2}+\omega_{3}^{2} e_{1}^{2} e_{2}^{2}\right)+\frac{23}{672}\left(\omega_{1}^{4}+\omega_{2}^{4}+\omega_{3}^{4}\right)\left(e_{1}^{2}+e_{2}^{2}+e_{3}^{2}\right)-\frac{5}{112}\left(e_{1}^{2} \omega_{1}^{4}+e_{2}^{2} \omega_{2}^{4}+e_{3}^{2} \omega_{3}^{4}\right)+\frac{1}{56}\left(e_{1}^{2}+e_{2}^{2}+e_{3}^{2}\right)\left(\omega_{1}^{2} \omega_{2}^{2}\right. \\
& \left.+\omega_{2}^{2} \omega_{3}^{2}+\omega_{3}^{2} \omega_{1}^{2}\right)+\frac{3}{56}\left(\omega_{1}^{2} \omega_{2}^{2} e_{3}^{2}+\omega_{2}^{2} \omega_{3}^{2} e_{1}^{2}+\omega_{3}^{2} \omega_{1}^{2} e_{2}^{2}\right)+\frac{7}{286}\left(e_{1}^{6}+e_{2}^{6}+e_{3}^{6}\right)-\frac{201}{2002} e_{1}^{2} e_{2}^{2} e_{3}^{2}
\end{aligned}
$$

where $\omega^{2}=\omega_{1}^{2}+\omega_{2}^{2}+\omega_{3}^{2}$. Results with $n=2,4$ agree with those of Siggia. ${ }^{31}$

\section{B. Strain rate and vorticity}

In order to calculate the averages of the above velocity derivatives, we need to determine the principal rates of strain $\left(e_{1}, e_{2}, e_{3}\right)$ in the vortex tubes and then perform volume averages; or equivalently using an ergodic hypothesis integrate over the vortex cross section and the lifetime of the vortex. We shall discuss in Sec. VI A the calculation of mean values of moments of the vorticity distribution. Here we give the expressions for the rates of strain in terms of the vorticity distribution of Eqs. (1)-(4). In terms of cy- 
lindrical polar velocity components $\left(u_{r} u_{\theta}, 0\right)$, which are independent of $z$,

$e_{r r}=\frac{\partial u_{r}}{\partial r}, \quad e_{r \theta}=\frac{1}{2} \frac{\partial u_{\theta}}{\partial r}-\frac{u_{\theta}}{2 r}+\frac{1}{2 r} \frac{\partial u_{r}}{\partial \theta}, \quad e_{\theta \theta}=\frac{1}{r} \frac{\partial u_{\theta}}{\partial \theta}+\frac{u_{r}}{r}$.

The other components are zero. The vorticity, whose only nonzero component is in the $z$ direction, is related to the velocity by

$$
\omega=\frac{\partial u_{\theta}}{\partial r}+\frac{u_{\theta}}{r}-\frac{1}{r} \frac{\partial u_{r}}{\partial \theta} .
$$

These are the rates of strain and vorticity associated with the stretched vortex. In addition, there is the strain due to the external stretching, with nonzero diagonal components $\left(-\frac{1}{2} a,-\frac{1}{2} a, a\right)$. The turns of the spiral become more and more circular as $\tau$ increases, in which case $u_{r} \ll u_{\theta}$ and $\partial / \partial r>1 / r$. It then follows from (56) and (57) that for the leading-order contributions,

$$
e_{r \theta}=\frac{1}{2} \omega, \quad e_{r r}=0, \quad e_{\theta \theta}=0 .
$$

This formula relies on the existence of large radial gradients on the vortex spiral and describes the contributions to the rate of strain from the asymmetric spiral turns. This is not the case for the contribution from the coarse grained, $\theta$-averaged contribution $\omega_{0}$ to the vorticity, for which

$$
e_{r \theta}=\frac{\omega_{0}}{2}-\frac{u_{0 \theta}}{r}, \text { where } u_{0 \theta}=\frac{1}{r} \int_{0}^{r} r \omega_{0} d r .
$$

The principal rates of strain to be inserted into the formulas in Sec. V A are

$$
e_{1}=-\frac{1}{2} a+e_{r \theta}, \quad e_{2}=-\frac{1}{2} a-e_{r \theta}, \quad e_{3}=a,
$$

where $e_{r \theta}$ is given by (58) or (59) for asymmetric or axisymmetric calculations, respectively.

A formal estimate of the various terms can be obtained as follows. Suppose, for simplicity, that viscous decay is neglected. Then for the nonaxisymmetric components,

$$
\begin{aligned}
& \omega^{\prime} \sim e^{a t} f(\rho) \sum^{\prime} e^{i n(\theta-\Omega \tau)}, \\
& \psi^{\prime} \sim \frac{f(\rho)}{\Lambda^{2} \tau^{2}} \sum \frac{e^{i n(\theta-\Omega \tau)}}{n^{2}}, \\
& u_{r}^{\prime} \sim \frac{i f(\rho) e^{a t / 2}}{\Lambda^{2} \tau^{2} \rho} \sum^{\prime} \frac{e^{i n(\theta-\Omega \tau)}}{n}, \\
& u_{\theta}^{\prime} \sim \frac{i f(\rho) e^{a t / 2}}{\Lambda \tau} \sum^{\prime} \frac{e^{i n(\theta-\Omega \tau)}}{n} .
\end{aligned}
$$

The prime denotes that the term with $n=0$ is omitted. It follows from (61) and (64) that

$$
e_{r \theta}^{\prime}=\frac{1}{2} \omega^{\prime}+O\left(\tau^{-1}\right) \text {. }
$$

See Appendix $\mathrm{C}$ for a discussion of the isotropy relation of the velocity gradients.

\section{A prediction}

Substituting (60) into (52b) and (52c) gives

$$
\begin{aligned}
& \left\langle\left(\frac{\partial u}{\partial x}\right)^{2}\right\rangle=\frac{3 a^{2}+4 e_{r \theta}^{2}}{15} \\
& \left\langle\left(\frac{\partial u}{\partial x}\right)^{3}\right\rangle=\frac{2 a\left(a^{2}-4 e_{r \theta}^{2}\right)}{35} .
\end{aligned}
$$

It will be shown later that $\omega^{2 p}, p \geqslant 2$, is proportional to the large quantity $R^{2} a / 4 v$ to a positive exponent. The dominant contributions to the velocity derivative moments in (52) for $n \geqslant 4$ may thus be obtained by substituting (60) with (65), and $\omega_{1}=\omega_{2}=0, \omega_{3}=\omega$ into $(52 \mathrm{c})-(52 \mathrm{k})$ and (55), and retaining in each equation only the term in the highest power of $\omega^{2}$. This gives

$$
\begin{aligned}
& \left\langle\left(\frac{\partial u}{\partial x}\right)^{2 p}\right\rangle=\hat{U}_{2 p} \omega^{2 p}, \quad\left\langle\left(\frac{\partial u}{\partial x}\right)^{2 p+1}\right\rangle=-a \hat{V}_{2 p} \omega^{2 p} \\
& \left\langle\left(\frac{\partial v}{\partial x}\right)^{2 p}\right\rangle=\hat{W}_{2 p} \omega^{2 p}, \quad p=2,3, \ldots
\end{aligned}
$$

where the numbers $\hat{U}_{2 p}$ and $\hat{V}_{2 p}$ are given by

$$
\begin{aligned}
& \hat{U}_{4}=\frac{1}{105}, \quad \hat{U}_{6}=\frac{5}{3003}, \quad \hat{U}_{8}=\frac{7}{21879}, \\
& \hat{U}_{10}=\frac{3}{46189}, \quad \hat{U}_{12}=\frac{33}{2414425}, \\
& \hat{V}_{5}=\frac{4}{231}, \quad \hat{V}_{7}=\frac{2}{429}, \quad \hat{V}_{9}=\frac{56}{46189}, \quad \hat{V}_{11}=\frac{30}{96577}, \\
& \hat{W}_{4}=\frac{2}{35}, \quad \hat{W}_{6}=\frac{100}{3003} .
\end{aligned}
$$

The approximation $a^{2}<\overline{\omega^{2}}$ is good since $\overline{\overline{\omega^{2}}}=\epsilon$ and $a^{2} v / \epsilon=0.0126$ from Table II.

We now define the generalized hyperflatness factors

$$
\begin{gathered}
F_{2 p}=\frac{\left\langle\overline{\left.(\partial u / \partial x)^{2 p}\right\rangle}\right.}{\left\langle(\partial u / \partial x)^{2}\right\rangle^{p}} \equiv \frac{\overline{\overline{(\partial u / \partial x)^{2 p}}}}{\left[\overline{\left.\overline{(\partial u / \partial x)^{2}}\right]^{p}}\right.}, \quad p=2,3, \ldots, \\
G_{2 p}=\frac{\overline{\left\langle(\partial v / \partial x)^{2 p}\right\rangle}}{\left\langle\overline{\left\langle(\partial u / \partial x)^{2}\right.}\right\rangle^{p}} \equiv \frac{\overline{(\partial v / \partial x)^{2 p}}}{\left[\overline{\left.\overline{(\partial u / \partial x)^{2}}\right]^{p}}\right.}, \quad p=2,3, \ldots ;
\end{gathered}
$$

the generalized hyperskewness factors

$$
\begin{aligned}
S_{2 p+1} & =\frac{\left.\overline{\left\langle(\partial u / \partial x)^{2 p+1}\right.}\right\rangle}{\left\langle\overline{(\partial u / \partial x)^{2}}\right\rangle^{(2 p+1) / 2}} \\
& =\frac{\overline{\overline{(\partial u / \partial x)^{2 p+1}}}}{\left.\overline{\left[(\partial u / \partial x)^{2}\right.}\right]^{(2 p+1) / 2}}, \quad p=2,3, \ldots ;
\end{aligned}
$$

and the vorticity moments

$$
\Omega_{2 p}=\frac{\left.\overline{\left\langle\omega_{x}^{2 p}\right.}\right\rangle}{\left\langle\overline{\omega_{x}^{2}}\right\rangle^{p}} \equiv \frac{\overline{\omega_{x}^{2 p}}}{\left.\overline{\left(\omega_{x}^{2}\right.}\right)^{p}}, \quad p=2,3, \ldots,
$$

where (50) has been used and where $\left\langle\omega_{x}^{2}\right\rangle=\omega^{2} / 3$.

When (67) are integrated over the vortex cross section and through the spiral structure lifetime (see Sec. VI for a 
TABLE III. Ratios of hyperskewness $S_{2 p+1}$ to hyperflatness $F_{2 p}, p$ $=2,3,4$. Values for $R_{\lambda}<82.9 ; 128^{3}$ numerical simulation Kerr. ${ }^{9} R_{\lambda}=150$, Vincent and Meneguzzi. ${ }^{14}$ Bracketed results were obtained by Vincent and Meneguzzi assuming exponential tails for the distribution of $\partial u / \partial x$ outside the range of the sample.

\begin{tabular}{lcccc}
\hline \hline & $\frac{S_{5}}{F_{4}}$ & $\frac{S_{7}}{F_{6}}$ & $\frac{S_{9}}{F_{8}}$ & $\frac{S_{11}}{F_{10}}$ \\
\hline$R_{\lambda}$ & -1.66 & -2.99 & & \\
37.2 & -1.65 & -2.92 & & \\
48.5 & -1.74 & -3.36 & & \\
55.9 & -1.74 & -3.32 & & \\
82.9 & -1.84 & -3.83 & & -8.99 \\
150 & -1.74 & -3.70 & -6.19 & $(-0.63)$ \\
150 & $(-1.65)$ & $(-2.56)$ & $(-1.79)$ & -2.1 \\
Present & -0.79 & -1.2 & -2.00 & \\
\hline \hline
\end{tabular}

discussion of this procedure), use is made of (42) and of the result $a^{2} v / \epsilon=$ const, it follows that

$$
\begin{aligned}
& \frac{S_{2 p+1}}{F_{2 p}}=\text { const }, \quad \frac{F_{2 p}}{\Omega_{2 p}}=\text { const }, \\
& \frac{G_{2 p}}{\Omega_{2 p}}=\text { const }, \quad p=2,3, \ldots,
\end{aligned}
$$

i.e., independent of $R_{\lambda}$. In particular

$$
\begin{aligned}
& \frac{S_{5}}{F_{4}}=-\frac{20}{11} \sqrt{15}\left(\frac{a^{2} v}{\epsilon}\right)^{1 / 2}, \quad \frac{S_{7}}{F_{6}}=-\frac{14}{5} \sqrt{15}\left(\frac{a^{2} v}{\epsilon}\right)^{1 / 2}, \\
& \frac{S_{9}}{F_{8}}=-\frac{88}{19} \sqrt{15}\left(\frac{a^{2} v}{\epsilon}\right)^{1 / 2}, \quad \frac{S_{11}}{F_{10}}=-\frac{110}{23} \sqrt{15}\left(\frac{a^{2} v}{\epsilon}\right)^{1 / 2} .
\end{aligned}
$$

Using $a^{2} v / \epsilon=0.0126$ then gives $S_{5} / F_{4}=-0.79$, $S_{7} / F_{7}=-1.2, S_{9} / F_{8}=-2.0$, and $S_{11} / F_{10}=-2.1$. Also, using (53),

$$
\frac{\Omega_{2 p}}{F_{2 p}}=\frac{1}{5^{p}(2 p+1)} \frac{\overline{\omega^{2 p}}}{\left\langle\overline{(\partial u / \partial x)^{2 p}}\right\rangle},
$$

and from (67) the right-hand side of this equation depends only on $p$. From (67) and (68) we find $\Omega_{4} / F_{4}=0.84$, $\Omega_{6} / F_{6}=0.69, \Omega_{8} / F_{8}=0.56$, and $\Omega_{10} / F_{10}=0.45$. Similar results can be obtained for the ratio $G_{4} / F_{4}$ and $G_{6} / F_{6}$.

In Tables III and IV these ratios are compared with results obtained from the the $128^{3}$ numerical simulations of Kerr ${ }^{9}$ at $R_{\lambda} \leqslant 82.9$ and the $240^{3}$ simulation of Vincent and Meneguzzi at $R_{\lambda}=150$. Kerr ${ }^{9}$ gives an extensive discussion of errors for the velocily-derivative moments. The ratio $S_{5} / F_{4}$ is approximately constant to within these error estimates but the numerical value of near -1.7 is larger than the present value of -0.79 . The same is true for the ratio $S_{7} / F_{6}$ and for $\Omega_{4} / F_{4}$ in Table IV. Simulation values of $\Omega_{6} / F_{6}$ may indicate an increase with $R_{\lambda}$ although errors in the numerical simulations are large for these quantities.

We comment that the result (75) and the present numerical values do not depend on the assumptions and re-
TABLE IV. Ratios of vorticity moment $\hat{\Omega}_{2 p}$ to hyperflatness $F_{2 p}, p$ $=2,3,4$. Values for $R_{\lambda}<82.9 ; 128^{3}$ numerical simulation Kerr. ${ }^{9}$

\begin{tabular}{llll}
\hline \hline$R_{\lambda}$ & $\frac{\hat{\Omega}_{4}}{F_{4}}$ & $\frac{\hat{\Omega}_{6}}{F_{6}}$ & $\frac{\hat{\Omega}_{8}}{F_{8}}$ \\
\hline 28.5 & 1.14 & 1.13 & 1.41 \\
37.2 & 1.18 & 1.52 & 2.04 \\
48.5 & 1.25 & 1.74 & 2.61 \\
55.9 & 1.26 & 1.80 & 2.72 \\
82.9 & 1.39 & 2.52 & 6.71 \\
Present & 0.84 & 0.69 & 0.56 \\
\hline \hline
\end{tabular}

sults of Secs. III and IV but follow from the large time behavior of the contracting spiral solution of the NavierStokes equations given by (1)-(4). Equations (73) and (74), however, depend on the assumptions of previous sections which lead to the result $a^{2} v / \epsilon=$ const.

\section{THE VORTICITY MOMENTS $\overline{\left\langle\omega^{2 p}\right\rangle}$}

\section{A. Cross section and time integration}

In axes fixed in a vortex tube we may put $\left(\omega_{1}, \omega_{2}, \omega_{3}\right)=(0,0, \omega)$ where $\omega$ is given by (1)-(4). The $2 p$ th moment of $\omega$ may then be written

$$
\begin{aligned}
\overline{\omega^{2 p}}= & N \int_{0}^{2 \pi} \int_{0}^{\infty} \int_{t_{1}}^{\infty} \exp (a t) \\
& \times\left(\sum_{n=-\infty}^{\infty} \omega_{n}(r, t) \exp (\operatorname{in} \theta)\right)^{2 p} d t r d r d \theta, \\
& p \geqslant 1,
\end{aligned}
$$

where $\omega_{n}(r, t)$ is given by (2). The $\exp (a t)$ factor is present owing to the axial lengthening of the vortex tube by the $z$ component of the stain rate. Equation (76) has been obtained by raising (1) to the power $2 p$ and integrating across the vortex cross section and in time from the cutoff time $t_{1}$ to $\infty$. The triple integral is the definition of the " operation. In stretched $(\rho, \tau)$ coordinates this becomes

$$
\begin{aligned}
\overline{\omega^{2 p}}= & N \int_{0}^{2 \pi} \int_{0}^{\infty} \int_{\phi / a}^{\infty}(1+a \tau)^{2 p-1} \\
& \times\left(\sum_{n=-\infty}^{\infty} \hat{\omega}_{n}(\rho, \tau) \exp (\operatorname{in} \theta)\right)^{2 p} d \tau \rho d \rho d \theta, \quad p \geqslant 1,
\end{aligned}
$$

$\hat{\omega}_{n}(\rho, \tau)=f(\rho) \exp \left[-i n \Omega(\rho) \tau-v n^{2} \Lambda^{2}(\rho) \tau^{3} / 3\right]$,

where the cutoff time is now expressed as $\tau_{1}=\phi / a$. When $\tau_{1}=0$ the integrals in general diverge owing to the singular character of (1)-(4) in this limit. Hence our results will depend on $\phi$ which will remain a free parameter but which, for reasons given earlier following (8), we expect to be $O(1)$. The case $p=1$ is special because it is related to the dissipation and will be discussed separately later. Expand- 
ing the Fourier series to the $2 p$ th power in (77) and performing the $\theta$ integration gives

$$
\begin{aligned}
\overline{\omega^{2 p}}= & 2 \pi N \int_{0}^{\infty} \int_{\phi / a}^{\infty}(1+a \tau)^{2 p-1} \\
& \times \sum \sum \cdots \sum \exp \left(-\frac{v J \Lambda^{2} \tau^{3}}{3}\right) f^{2 p}(\rho) \\
& \sum \begin{array}{c}
i_{1}, i_{2}, \ldots, i_{2 p} \neq 0 \\
i_{1}+i_{2}+\cdots+i_{2 p}=0 \\
\end{array} \\
\times & d \tau \rho d \rho+\text { Core }, \quad p \geqslant 2,
\end{aligned}
$$

where $J=i_{1}^{2}+i_{2}^{2}+\cdots+i_{2 p}^{2}$. The "Core" corresponds to the strictly axisymmetric contribution $i_{1}, i_{2}, \ldots, i_{2 p}=0$ which will later be shown to be small compared to the retained terms.

The $2 p$-fold sum in (79) is over the lattice of integers $i_{1}, i_{2}, \ldots, i_{2 p}$ which lie on the hyperplane $i_{1}+i_{2}+\cdots+i_{2 p}=0$. By use of the Dirichlet integrals (Jeffreys and Jeffreys, ${ }^{32}$ p. 468) this sum can be approximated by an integral over the surface of this hyperplane as

$$
\begin{aligned}
& \sum \sum \cdots \sum\{\cdots\} \rightarrow A_{2 p} \int_{0}^{\infty}\{\cdots\} J^{p-3 / 2} d J, \\
& i_{1}+i_{1}+\cdots+i_{2}, \cdots, i_{2 p} \neq 0 \\
& A_{2 p}=\frac{\pi_{2 p}=0}{\sqrt{2 p} \Gamma[p-1 / 2]},
\end{aligned}
$$

where the $\{\cdots\}$ refers to any implicit or explicit function of $J$, and $A_{2 p}$ is related to the volume of a $(2 p-1)$ dimensional hypersphere of unit radius. Using (80), (79) can be then approximated as

$$
\begin{aligned}
\overline{\omega^{2 p}}= & 2^{2 p} \pi N A_{2 p} \int_{0}^{\infty} f^{2 p}(\rho) \\
& \times \int_{\phi / a}^{\infty} \int_{0}^{\infty}(1+a \tau)^{2 p-1}(4 J)^{p-(3 / 2)} \\
& \times \exp \left(-\frac{4}{3} v J \Lambda^{2}(\rho) \tau^{3}\right) d J d \tau \rho d \rho, \quad p \geqslant 2 .
\end{aligned}
$$

The double integral with respect to $J$ and $\tau$ can be evaluated by first expanding the $(1+a \tau)^{2 p-1}$ factor with the binomial theorem, second, by making a change of integration variable with $u=\frac{4}{3} v J \Lambda^{2}(\rho) \tau^{3}$, and finally by exchanging the limits of integration in the $(J-u)$ plane. After some straightforward but tedious algebra we obtain

$$
\begin{aligned}
\overline{\omega^{2 p}}=\frac{N a^{3 p-5 / 2}}{v^{p-1 / 2}} \hat{L}_{2 p}(\phi) \int_{0}^{\infty} \frac{f^{2 p}(\rho) \rho d \rho}{|\Lambda(\rho)|^{2 p-1}}, \quad p>2, \\
\hat{L}_{2 p}(\phi)= \\
\quad \times \sum_{q=0}^{2 p-1} \frac{\phi^{q+5 / 2-3 p}}{\sqrt{p}} \frac{\Gamma[2 p](3 \pi)^{p-1 / 2}}{(6 p-2 q-5) \Gamma[q+1] \Gamma[2 p-q]} .
\end{aligned}
$$

When additional functions describing the initial conditions are required, for example $M$ functions $f_{m}(\rho), m=1, ., M$ describing $M$ interleaved spirals with arbitrary phases, the integral in (82a) is replaced by

$$
\sum_{m=1}^{M} \int_{0}^{\infty} \frac{\left|f_{m}(\rho)\right|^{2 p} \rho d \rho}{|\Lambda(\rho)|^{2 p-1}}
$$

Using (20) and (21) and rearranging, (82a) may be written as

$$
\begin{gathered}
\overline{\omega^{2 p}}=2^{2 p-3} a^{2 p} \hat{L}_{2 p}(\phi) \hat{K}_{2 p}\left(\frac{N R^{2}}{a}\right)\left(\frac{\Gamma_{0}}{v}\right)\left(\frac{a^{2} R}{4 v}\right)^{p-3 / 2}, \\
p \geqslant 2 \\
\hat{K}_{2 p}=\int_{0}^{\infty} \frac{\widetilde{f}^{2 p}(\xi) \xi d \xi}{|\hat{\Lambda}(\xi)|^{2 p-1}}
\end{gathered}
$$

where $\tilde{f}(\xi)$ is given by $(22)$. The quantities $\hat{L}_{2 p}(\phi)$ and $\hat{K}_{2 p}$ are pure numbers with $\hat{L}_{2 p}(\phi) \rightarrow \infty, \phi \rightarrow 0$. Note that the passage from (82) to (84) introduces a dependence on the Reynolds number parameter $a R^{2} / 4 v$. Use of (53), (72), and the standard result for isotropic turbulence, $\epsilon$ $=3 v \overline{\overline{\omega_{x}^{2}}}$, then gives

$$
\begin{aligned}
\Omega_{2 p}= & \frac{2^{2 p-3} 3^{p} \hat{L}_{2 p}(\phi) \hat{K}_{2 p}}{2 p+1}\left(\frac{N R^{2}}{a}\right)\left(\frac{\Gamma_{0}}{v}\right) \\
& \times\left(\frac{a^{2} v}{\epsilon}\right)^{p}\left(\frac{a R^{2}}{4 v}\right)^{p-3 / 2}, \quad p \geqslant 2 .
\end{aligned}
$$

For $p \geqslant 2, \Omega_{2 p}$ exhibits power-law behavior in $a R^{2} / v$ with positive exponent.

\section{B. Contribution of the core}

The contribution of the core to $\overline{\omega^{2 p}}$ in (76) can be written

$\overline{\omega_{0}^{2 p}}=2 \pi N \int_{0}^{\infty} \int_{\phi / a}^{\infty}(1+a \tau)^{2 p-1} \hat{\omega}_{0}^{2 p}(\rho, \tau) d \tau \rho d \rho, \quad p \geqslant 1$.

In (86) $\hat{\omega}_{0}(\rho, \tau)$ is the solution of the heat equation in the stretched coordinates $(\rho, \tau)$ with initial conditions given by the $\theta$-averaged vorticity $f(\rho)+g(\rho)$. From (30) this solution is

$$
\begin{aligned}
\hat{\omega}_{0}(r, t)= & \frac{1}{2 v \tau} \exp \left(-\frac{\rho^{2}}{4 v \tau}\right) \int_{0}^{\infty}[f(u)+g(u)] \\
& \times \exp \left(-\frac{u^{2}}{4 v \tau}\right) I_{0}\left(\frac{\rho u}{2 v \tau}\right) u d u .
\end{aligned}
$$

Using (20)-(23) this may be written as

$$
\hat{\omega}_{0}(r, t)=a\left(\frac{\Gamma_{0}}{v}\right) S\left(\xi, v ; \widetilde{R}^{2}\right),
$$




$$
\begin{aligned}
S\left(\xi, v ; \widetilde{R}^{2}\right)= & \frac{1}{2 v} \exp \left(-\frac{\widetilde{R}^{2} \xi^{2}}{v}\right) \int_{0}^{\infty}[\tilde{f}(\zeta)+\widetilde{g}(\zeta)] \\
& \times \exp \left(-\frac{\widetilde{R}^{2} \xi^{2}}{v}\right) I_{0}\left(\frac{2 \tilde{R}^{2} \xi \zeta}{v}\right) \zeta d \xi
\end{aligned}
$$

where $v=a \tau$ and $\widetilde{R}^{2}=a R^{2} / 4 v$. When $\widetilde{f}(\xi), \widetilde{g}(\xi)$ are given by (22) and (23) the integral in (88b) can be evaluated in closed form in terms of the hypergeometric function but the result is cumbersome and is not reproduced here. Substituting this equation into (86) and using arguments paralleling those leading to (85) then gives the core contribution to $\Omega_{0_{2 p}}$ as

$$
\begin{aligned}
& \Omega_{0_{2 p}}=\frac{2 \pi 3^{p}}{2 p+1} \hat{C}_{2 p}\left(\phi, \widetilde{R}^{2}\right)\left(\frac{N R^{2}}{a}\right)\left(\frac{\Gamma_{0}}{v}\right)^{2 p}\left(\frac{a^{2} v}{\epsilon}\right)^{p}, \quad p \geqslant 2, \\
& \hat{C}_{2 p}\left(\phi, \widetilde{R}^{2}\right)=\int_{0}^{\infty} \int_{\phi / a}^{\infty}(1+v)^{2 p-1} S^{2 p}\left(\xi, v ; \widetilde{R}^{2}\right) \xi d \xi d v .
\end{aligned}
$$

Numerical evaluation of $\hat{C}_{2 p}\left(\phi, \widetilde{R}^{2}\right)$ for $\phi=1$ shows very weak and monotonically decreasing dependence on $\widetilde{R}^{2}$. We find $\hat{C}_{4}(1,100) \approx 3 \times 10^{-7}, \hat{C}_{6}(1,100) \approx 3 \times 10^{-10}$, and $\hat{C}_{8}(1,100) \approx 3 \times 10^{-13}$. With $N R^{2} / a=0.1, \Gamma_{0} / \nu=200$, and $a^{2} v / \epsilon=0.0126$ from Sec. IV B, we find $\Omega_{0_{2 p}}<1$ for $p=2,3,4$ which will be seen to be small compared to contributions from the spiral given by (85).

\section{The special case $p=1$}

The dissipation $\epsilon$ can be evaluated in several ways:

(1) from (37); $p=1$

(2) using $\epsilon=v \omega^{2}$ where $\overline{\omega^{2}}$ is given by (77) with

(3) from $\epsilon=15 v \overline{\left\langle(\partial u / \partial x)^{2}\right\rangle}$ using (65) and (66a). For self-consistency these must all be in agreement. The formal equivalence of (37) and $\epsilon=\nu \overline{\omega^{2}}$ can be established for general $\omega(r, \theta, t)$ in the tube cross section by substitution of (11) and (12) into (37) and use of the result

$$
\int_{0}^{\infty} k J_{n}\left(k r_{1}\right) J_{n}\left(k r_{2}\right) d k=\frac{\delta\left(\dot{r}_{2}-r_{1}\right)}{\sqrt{r_{2} r_{1}}} .
$$

Since approximations have been made in obtaining the spiral spectrum in the form (14) and (15), it is useful to compare calculation of $\epsilon$ for the spiral obtained from substitution of (14) and (15) into the third term of (37) with that calculated from (77) and (78) with $p=1$ as

$$
\begin{aligned}
\epsilon_{0}+\epsilon_{s}= & 2 \pi \nu N \int_{0}^{\infty} \int_{\phi / a}^{\infty}(1+a \tau)\left(\hat{\omega}_{0}^{2}(\rho, \tau)\right. \\
& \left.+2 \sum_{n=1}^{\infty} \hat{\omega}_{n}^{2}(\rho, \tau)\right) d \tau \rho d \rho
\end{aligned}
$$

In (91), $\epsilon_{0}$ and $\epsilon_{s}$ refer to the dissipation in the core and in the spiral, respectively. Considering $\epsilon_{s}$ alone, using (78), and making the change of variables $u=n^{2} C \tau^{3}$ gives

$$
\begin{aligned}
\epsilon_{s}= & \frac{4 \pi v N}{3} \sum_{n=1}^{\infty} \int_{0}^{\infty} \int_{n^{2} C \phi^{3} / a^{3}}^{\infty}|f(\rho)|^{2} \\
& \times\left(C^{-1 / 3} n^{-2 / 3} u^{-2 / 3}\right. \\
& \left.+a C^{-2 / 3} n^{-4 / 3} u^{-1 / 3}\right) e^{-u} d u \rho d \rho,
\end{aligned}
$$

where $C=2 v \Lambda^{2}(\rho) / 3$. Designating the contributions to $\epsilon_{s}$ from the first and second terms in (92) by $\epsilon_{s_{1}}$ and $\epsilon_{s_{2}}$, respectively, it may be seen that the major contribution to $\epsilon_{s_{2}}$ comes from small $n$ when $C \phi^{3} / a^{3}$ is small $\left[C \phi^{3} / a^{3} \propto\left(\Gamma_{0} / v\right)^{2}\left(R^{2} a / 4 v\right)^{-3}\right.$ and is small for large $\left.R^{2} a / 4 v\right]$. Also, unlike the sum in $\epsilon_{s_{1}}$ and the $2 p$-fold sums in (79), the series $\Sigma n^{-4 / 3}$ is convergent at $\infty$; it is this that makes $p=1$ a special case. Hence we can put the lower limit of the $u$ integral in $\epsilon_{s_{2}}$ equal to zero and obtain

$$
\begin{aligned}
\epsilon_{s_{2}}= & \frac{4 \pi}{3}\left(\frac{3}{2}\right)^{2 / 3} \Gamma\left[\frac{2}{3}\right] N a v^{1 / 3} \sum_{n=1}^{\infty} n^{-4 / 3} \\
& \times \int_{0}^{\infty} \frac{|f(\rho)|^{2} \rho d \rho}{|\Lambda(\rho)|^{4 / 3}} .
\end{aligned}
$$

This agrees with the result obtained when (14) and (15) are substituted into the second integral of (37).

The term giving $\epsilon_{s_{1}}$ in (93) may be approximated as was done for the $\overline{\omega^{2 p}}$ integrals by replacing the sum by an integral and evaluating a resulting double integral by exchange of limits to give

$$
\epsilon_{s_{1}}=\frac{4 \pi}{\phi^{1 / 2}}\left(\frac{3}{2}\right)^{1 / 2} \Gamma\left[\frac{1}{2}\right] N a^{1 / 2} v^{1 / 2} \int_{0}^{\infty} \frac{|f(\rho)|^{2} \rho d \rho}{|\Lambda(\rho)|} .
$$

The ratio of $\epsilon_{s_{2}}$ to $\epsilon_{s_{1}}$ is

$$
\frac{\epsilon_{s_{1}}}{\epsilon_{s_{2}}} \propto \frac{1}{\phi^{1 / 2}}\left(\frac{\Gamma_{0}}{v}\right)^{1 / 3}\left(\frac{a R^{2}}{4 v}\right)^{-1 / 2} .
$$

This quantity is divergent when $\phi \rightarrow 0$ but if $\phi=O(1)$ and $a R^{2} / 4 v$ is large, the spiral dissipation is dominated by $\epsilon_{s_{2}}$ in agreement with the spectral calculation.

Use of $\epsilon=15 v \overline{\left\langle(\partial u / \partial x)^{2}\right\rangle}$ together with (65) and (66a) leads to a result identical to (91). Likewise when the skewness $S_{3}$ is calculated directly using (66b), (65), (77) and (78), a result is obtained which agrees with that found when (14) and (15) are used in the spectral term of (40).

\section{The vortex lateral scale $R$}

In order to compare calculations for velocityderivative and vorticity moments with experiment and computation it is necessary to relate the parameters of the 
TABLE V. Dimensionless coefficients $\hat{K}_{2 p}, \hat{L}_{2 p}$, and $\hat{\Omega}_{2 p}, \alpha=-0.5$, $\Gamma_{0} / \nu=100, N R^{2} / a=0.6$, and $\phi=0.475$.

\begin{tabular}{rccl}
\hline \multicolumn{1}{c}{$2 p$} & $\hat{K}_{2 p}$ & $\hat{L}_{2 p}$ & $\hat{\Omega}_{2 p}$ \\
\hline 4 & $4.04 \times 10^{-2}$ & $1.87 \times 10^{3}$ & 1.54 \\
6 & $1.97 \times 10^{-2}$ & $1.33 \times 10^{5}$ & 1.94 \\
8 & $1.02 \times 10^{-2}$ & $1.46 \times 10^{7}$ & 4.37 \\
10 & $5.51 \times 10^{-3}$ & $1.87 \times 10^{9}$ & $1.26 \times 10^{1}$ \\
12 & $3.04 \times 10^{-3}$ & $2.60 \times 10^{11}$ & $4.17 \times 10^{1}$ \\
16 & $9.70 \times 10^{-4}$ & $5.9 \times 10^{13}$ & $5.96 \times 10^{2}$ \\
20 & $3.2 \times 10^{-4}$ & $1.5 \times 10^{20}$ & $1.05 \times 10^{5}$ \\
40 & $1.6 \times 10^{-6}$ & $3.3 \times 10^{42}$ & $6.9 \times 10^{10}$ \\
60 & $9.9 \times 10^{-9}$ & $1.2 \times 10^{65}$ & $1.1 \times 10^{18}$ \\
100 & $3.7 \times 10^{-13}$ & $2.3 \times 10^{110}$ & $7.3 \times 10^{32}$ \\
\hline \hline
\end{tabular}

model to the microscale Taylor-Reynolds number $R_{\lambda}$. The present model, however, does not describe the energy containing range of eddies and so contains no information on the turbulence energy $\overline{\overline{u_{i}^{2}}} / 2\left[\left(u_{1}, u_{2}, u_{3}\right)\right.$ are the instantaneous velocity components] necessary to define $R_{\lambda}$. We thus introduce the assumption that

$$
R^{2}=\lambda\left(\frac{4 v}{a}\right)^{1 / 2}
$$

This asserts that $R$ is the geometric mean of the Taylor microscale $\lambda$ and the smallest physical scale in the model $(v / a)^{1 / 2}$. Equation (96) is motivated primarily by the observations of Vincent and Meneguzzi ${ }^{14}$ and Jiménez ${ }^{7}$ that the diameters of vortex tubes present in numerical simulations of isotropic turbulence are intermediate between the dissipation scale and $\lambda$. Use of (96) together with the standard definition of $R_{\lambda}$ from isotropic turbulence theory

$$
R_{\lambda} \equiv \frac{u \lambda}{v}, \quad \epsilon=\frac{15 v u^{2}}{\lambda^{2}}
$$

where $u=\left(\overline{\overline{u_{i}^{2}}}\right)^{1 / 2}$, is sufficient to obtain the relation

$$
\frac{a R^{2}}{4 v}=\left(\frac{15}{16}\right)^{1 / 4}\left(\frac{a^{2} v}{\epsilon}\right)^{1 / 4} R_{\lambda}^{1 / 2}
$$

Use of (98) in (85) then gives

$$
\begin{aligned}
\Omega_{2 p}= & \hat{\Omega}_{2 p} R_{\lambda}^{p / 2-3 / 4} \\
\hat{\Omega}_{2 p}= & \frac{2^{2 p-3} 3^{p} \hat{L}_{2 p}(\phi) \hat{K}_{2 p}}{2 p+1}\left(\frac{15}{16}\right)^{p / 4-3 / 8}\left(\frac{N R^{2}}{a}\right)\left(\frac{\Gamma_{0}}{v}\right) \\
& \times\left(\frac{a^{2} v}{\epsilon}\right)^{5 p / 4-3 / 8}, \quad p \geqslant 2
\end{aligned}
$$

Table $\mathrm{V}$ shows numerical values for the numbers $\hat{K}_{2 p}$ given by $(84 \mathrm{~b}), \hat{L}_{2 p}(\phi)$ given by $(82 \mathrm{~b})$, and $\hat{\Omega}_{2 p}$ defined by (99b). The first of these quantities is a function of $p$ only

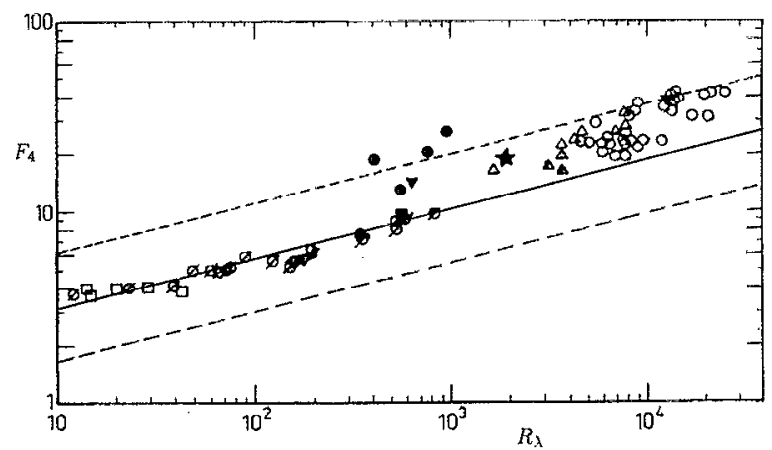

FIG. 4. Flatness factor $F_{4}$ vs $R_{\lambda} . \cdots: \phi=0.35$; $-:$ : $\phi=0.475 ;-\cdots$ : $\phi=0.7$. Symbols are a compilation of experimental data, Van Atta and Antonia ${ }^{19}$ (reproduced with permission).

while $\hat{L}_{2 p}$ and $\hat{\Omega}_{2 p}$ are functions of $\phi$ also. For reasons to be discussed, numerical values are tabulated for $\phi=0.475$.

\section{COMPARISON WITH EXPERIMENT AND NUMERICAL SIMULATION}

We now compare present calculated higher-order statistics with experiment and with the results of numerical simulation. We first discuss the hyperflatness factors $F_{2 p}$, $F_{2 p}$ and hyperskewness factors $S_{2 p+1}$ defined by (69)(71), respectively. Using the relation between the longitudinal velocity-derivative moments and the vorticity moments given by (67) and (68), $\overline{\omega_{2 p}}$ given by (84a), $\left\langle\overline{(\partial u / \partial x)^{2}}\right\rangle$ from $(42)$, and $a R^{2} / 4 v$ given by (98), we obtain after some manipulation

$$
\begin{aligned}
& F_{2 p}=\hat{F}_{2 p} R_{\lambda}^{p / 2-3 / 4}, \quad S_{2 p+1}=-\hat{S}_{2 p+1} R_{\lambda}^{p / 2-3 / 4} \\
& G_{2 p}=\hat{G}_{2 p} R_{\lambda}^{p / 2-3 / 4} \\
& \hat{F}_{2 p}=\hat{U}_{2 p} 5^{p}(2 p+1) \hat{\Omega}_{2 p}, \\
& \hat{S}_{2 p+1}=\hat{V}_{2 p} 5^{p} \sqrt{15}(2 p+1)\left(\frac{a^{2} v}{\epsilon}\right)^{1 / 2} \hat{\Omega}_{2 p} \\
& \hat{G}_{2 p}=\hat{W}_{2 p} 5^{p}(2 p+1) \hat{\Omega}_{2 p}, \quad p=2,3, \ldots
\end{aligned}
$$

where $\hat{U}_{2 p}, \hat{V}_{2 p}$, and $\hat{W}_{2 p}$ are given by $(68 \mathrm{a})-(68 \mathrm{c})$. With our preferred values $\alpha=-0.5, \Gamma_{0} / v=100, N R^{2} / a=0.6$, and $a^{2} v / \epsilon=0.0126$, the numbers $\hat{F}_{2 p}, \hat{S}_{2 p+1}, \hat{G}_{2 p}$, and $\hat{\Omega}_{2 p}$ are then functions of $p$ and $\phi$. We first consider $p=2$ for which $F_{4}, S_{5}, G_{4}$, and $\Omega_{4}$ are all proportional to $R_{\lambda}^{1 / 4}$, and choose $\phi=0.35,0.475$, and 0.70 We note that the exponent $1 / 4$ for $p=2$ is effectively fixed by the assumption given by (96): a different assumption for the relation of $R$ to the model parameters would give a different $R_{\lambda}$ exponent for all $p$. The present results for $p=2$ calculated from (99) to (100) are depicted in Figs. 4-7. In Fig. 4 the flatness factor $F_{4}$ is compared with experimental measurements compiled 


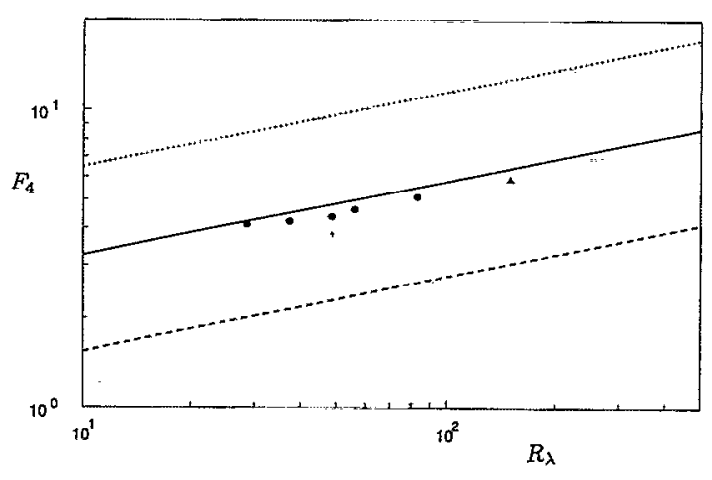

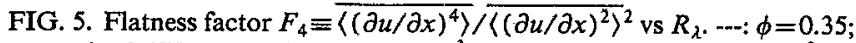
- $\phi=0.475 ;---; \phi=0.7 ; 0: 128^{3}$ numerical simulation, Kerr; ${ }^{9} \Delta$ : $240^{3}$ numerical simulation, Vincent and Meneguzzi. ${ }^{14}$

by Van Atta and Antonia ${ }^{19}$ from a variety of sources, including at large $R_{\lambda}$, atmospheric data. The best agreement with measurement is obtained with $\phi=0.475$ and we will view the comparison on Fig. 4 as effectively fixing this numerical value for $\phi$. Comparison of $F_{4}$ in Fig. 5 with the numerical simulations of $\mathrm{Kerr}^{9}$ and Vincent and Meneguzzi ${ }^{14}$ also indicates best agreement with $\phi=0.475$.

In Figs. 6 and 7 the present calculation with $\phi=0.475$ underpredicts the magnitudes of $S_{5}$ and $\Omega_{4}$ but the variation with $R_{\lambda}$, which in the model is independent of $\phi$, is in fair agreement with the simulations. Table $\mathrm{V}$ displays the variation of the numbers $\hat{\Omega}_{2 p}$ with $p(\phi=0.475)$ while Table VI shows computed $\hat{F}_{2 p}, \hat{S}_{2 p+1}$, and $\hat{G}_{2 p}$.

Table VII shows $F_{6}, S_{7}$, and $F_{8}(\phi=0.475)$ compared with simulation at various $R_{\lambda}$ while Table VIII shows a similar comparison for $\Omega_{6}$ and $\Omega_{8}$. It seems clear that the agreement with the simulations is better for the $F_{2 p}$ than for the $S_{2 p+1}$. Figures 4-7 indicate the sensitivity of the calculations to $\phi$ when the parameters $\alpha, \Gamma_{0} / v$, and $N R^{2} / a$. are fixed. However, when these parameters are chosen to give the Kolmogorov constant $\mathscr{K}_{0}$ and the skewness $S_{3}$ near to experimental values, and $\phi$ is further chosen to match $F_{4}$, the results for the higher-order velocity-

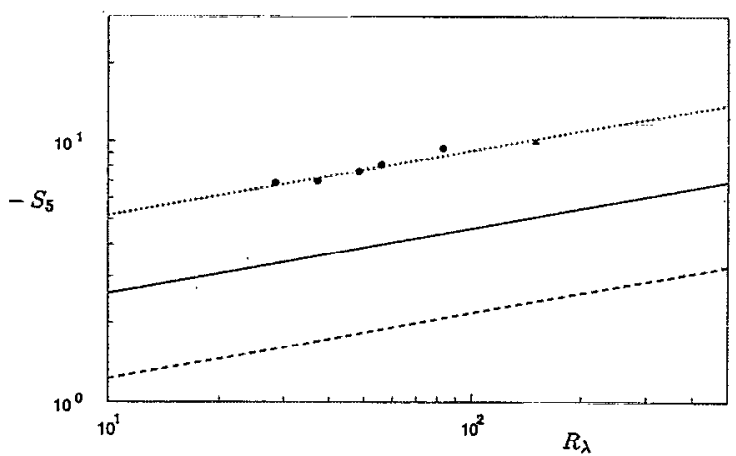

FIG. 6. Hyperskewness factor $S_{5} \equiv \overline{\left\langle(\partial u / \partial x)^{5}\right\rangle} / \overline{\left((\partial u / \partial x)^{2}\right)^{5 / 2}}$ vs $R_{\lambda} \ldots$ $\phi=0.35 ;-; \phi=0.475 ;---: \phi=0.7 ; 128^{3}$ numerical simulation, Kerr; ${ }^{9} \Delta: 240^{3}$ numerical simulation, Vincent and Meneguzzi. ${ }^{14}$

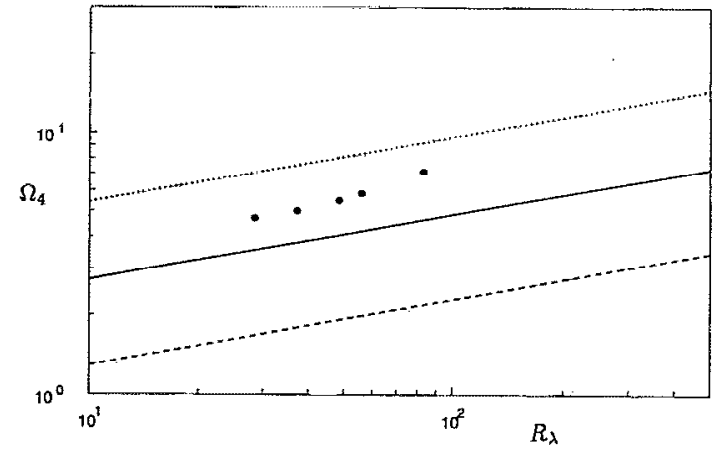

FIG. 7. Fourth-order vorticity moment $\Omega_{4}=\overline{\left\langle\omega_{x}^{4}\right\rangle} / \overline{\left\langle\omega_{x}^{2}\right\rangle^{2}}$ vs $R_{\lambda}$. $\phi=0.35$; - $\phi=0.475 ;---: \phi=0.7 ; \bigcirc 128^{3}$ numerical simulation, Kerr. $^{9}$

derivative moments are in reasonable agreement. This is illustrated in Table IX which shows results, designated as column B, obtained using $\alpha=-0.25, \Gamma_{0} / v=200$, $N R^{2} / a=0.2$ (for the corresponding $\mathscr{K}_{0}$ and $S_{3}$, see Table II), and $\phi=0.775$.

Kerr calculates the fourth-order velocity-derivative moments in the form of dimensionless forms of the four terms on the right-hand side of the fourth-order lateral velocity derivative, Eq. (55b). Wc denote these quantities by

$$
\begin{aligned}
& Q_{1}=\frac{15}{7} \frac{\overline{\left(e_{1}^{2}+e_{2}^{2}+e_{3}^{2}\right)^{2}}}{\overline{\left(e_{1}^{2}+e_{2}^{2}+e_{3}^{2}\right)^{2}}}, \quad Q_{2}=3 \frac{\overline{\omega^{2}\left(e_{1}^{2}+e_{2}^{2}+e_{3}^{2}\right)}}{\overline{\omega^{2}} \overline{\left(e_{1}^{2}+e_{2}^{2}+e_{3}^{2}\right)}}, \\
& Q_{3}=3 \frac{\overline{\left(\omega_{1}^{2} e_{1}^{2}+\omega_{2}^{2} e_{2}^{2}+\omega_{1}^{3} e_{3}^{2}\right)}}{\overline{\omega^{2}} \overline{\left(e_{1}^{2}+e_{2}^{2}+e_{3}^{2}\right)}}, \quad Q_{4}=3 \frac{\overline{\left(\omega_{1}^{4}\right)}}{\left(\overline{\omega^{2}}\right)^{2}} .
\end{aligned}
$$

From (60) and (65) and using $\left(\omega_{1}, \omega_{2}, \omega_{3}\right)=(0,0, \omega)$ in the reference frame where the three axes are aligned with the vortex tube it follows that to leading order $Q_{1}, Q_{2}$, and $Q_{4}$ are all proportional to $\overline{\omega^{4}}$ and therefore to $\Omega_{4}$ with equality in the case of $Q_{4}$. Hence in the present model these quantities are in turn proportional to $R_{\lambda}^{1 / 4}$. Putting $Q_{i}=\hat{Q}_{i} R_{\lambda}^{1 / 4}$, $i=1,2$, a simple calculation shows that $\hat{Q}_{1}=25 \hat{\Omega}_{4} / 21$ and $\hat{Q}_{2}=5 \hat{\Omega}_{4} / 3$. Numerical values of $Q_{1}$ and $Q_{2}$ are compared with simulation results obtained by $\mathrm{Kerr}^{9}$ in Table X where it is seen that these quantities are similar in magnitude, and, from Fig. 7 , similar in magnitude to $\Omega_{4}$. Since in the model the vorticity is aligned with the principal rate of strain $e_{3}=a$, the numerator in the first equation of (101b) is proportional to $a^{2} \omega^{2}$. A short calculation then gives

TABLE VI. Numbers $\hat{F}_{2 p}, \hat{S}_{2 p+1}, \hat{G}_{2 p}$ vs $p, \alpha=-0.5, \Gamma_{0} / y=100$, $N R^{2} / a=0.6$, and $\phi=0.475$.

\begin{tabular}{clll}
\hline \hline$p$ & \multicolumn{1}{c}{$\hat{F}_{2 p}$} & \multicolumn{1}{c}{$\hat{S}_{2 p+1}$} & \multicolumn{1}{c}{$\hat{G}_{2 p}$} \\
\hline 2 & 1.83 & 1.46 & $1.10 \times 10^{1}$ \\
3 & 2.84 & 3.48 & $5.68 \times 10^{1}$ \\
4 & 7.87 & $1.31 \times 10^{1}$ & \\
5 & $2.81 \times 10^{1}$ & $5.88 \times 10^{1}$ & \\
6 & $1.16 \times 10^{2}$ & & \\
\hline \hline
\end{tabular}


TABLE VII. $F_{6}, S_{7}$, and $F_{8}$ vs $R_{\lambda}$. Left-hand entries are numerical simulation; $R_{\lambda}<82.9$; $128^{3}$, Kerr. ${ }^{9} R_{\lambda}=150$; Vincent and Meneguzzi, ${ }^{14} 240^{3}$. Bracketed results obtained by assuming exponential tails for the distribution of $\partial u / \partial x$ outside the range of the sample. Right-hand entries are present results for $\alpha=-0.5, \Gamma_{0} / \nu=100, N R^{2} / a=0.6$, and $\phi=0.475$.

\begin{tabular}{rrrrrrr}
\hline \multicolumn{1}{c}{$R_{\lambda}$} & \multicolumn{2}{c}{$F_{6}$} & \multicolumn{2}{c}{$S_{7}$} & \multicolumn{2}{c}{$F_{8}$} \\
\hline 28.5 & 39.5 & 35.0 & -118 & -43.0 & 701 & 519 \\
37.2 & 40.1 & 42.7 & -117 & -52.5 & 638 & 723 \\
48.5 & 47.2 & 52.1 & -159 & -64.0 & 1003 & 1008 \\
55.9 & 53.8 & 58.0 & -179 & -71.2 & 1206 & 1203 \\
82.9 & 73.3 & 78.0 & -281 & -95.7 & 2326 & 1970 \\
150.0 & 104.9 & 122.0 & -389 & -149.0 & 4344 & 4130 \\
150.0 & $(131.9)$ & & $(-339)$ & & $(9362)$ \\
\hline
\end{tabular}

$Q_{3}=6\left(a^{2} v / \epsilon\right)$ which is constant independent of $R_{\lambda}$. With $a^{2} v / \epsilon=0.0126$ we obtain $Q_{3} \approx 0.07$ compared to the nearly constant value found by Kerr of 0.7 . Kerr observes that this low value of $Q_{3}$ in comparison with $Q_{1}, Q_{2}$, and $Q_{4}$ suggests that the vorticity vector is preferentially nearly perpendicular to the directions of the principal rates of strain of largest magnitude. Since presently $a \leqslant\left(\overline{\omega^{2}}\right)^{1 / 2}$ then from (60) and (65) this is a property of the present model. Indeed if in (60) and (65) we estimate $\omega^{\prime}$ $\approx\left(\bar{\omega}^{2}\right)^{1 / 2} \approx(\epsilon / \nu)^{1 / 2}$ together with $a(v / \epsilon)^{1 / 2} \approx 1 / 9$ we can estimate $e_{1} / a: e_{2} / a: e_{3} / a \approx 4:--5: 1$ with the intermediate principal rate of strain aligned with the vorticity vector. This agrees approximately with Ashurst's ${ }^{6}$ estimate $3:-4: 1$ based on the Burgers vortex with $\Gamma_{0} / v \approx 300$.

It has been pointed out to us by Dimotakis ${ }^{33}$ that owing to long tails in the probability distributions for $\omega_{1}$ and the longitudinal velocity derivatives, high-order moments are extremely difficult to obtain accurately in either simulation or experiment. This is illustrated in Table IX where hyperflatness and hyperskewness factors $\overline{\left\langle(\partial u / \partial x)^{n}\right\rangle} / \overline{\left\langle(\partial u / \partial x)^{2}\right\rangle^{n / 2}}, n=4,5, \ldots, 12$ are compared with unpublished results supplied by Meneguzzi ${ }^{34}$ and obtained from the $240^{3}$ simulations of Vincent and Meneguzzi. ${ }^{14}$ Two sets of simulation results are shown, each obtained from a sample size of order $10^{9}$, the first direct from the numerical sample and the second assuming exponential tails on the distribution of $\partial u / \partial x$ outside the range of the data. The differences between our predictions and the simulation results is generally of the same order of magnitude as the differences between the simulation results obtained with and without assumed tails. Finally for $G_{4}$

TABLE VIII. $\Omega_{6}$ and $\Omega_{8}$ vs $R_{\lambda}$. Left-hand entries are numerical simulation; $128^{3}$, Kerr. ${ }^{9}$ Right-hand entries are present results for $\alpha=-0.5$, $\Gamma_{0} / \nu=100, N R^{2} / a=0.6$, and $\phi=0.475$.

\begin{tabular}{crrrr}
\hline \hline$R_{\lambda}$ & \multicolumn{3}{c}{$\Omega_{6}$} & \multicolumn{2}{c}{$\Omega_{8}$} \\
\hline 28.5 & 51.8 & 23.9 & 988 & 288 \\
37.2 & 61.2 & 29.2 & 1302 & 401 \\
48.5 & 82.3 & 35.6 & 2620 & 559 \\
55.9 & 96.7 & 39.7 & 3284 & 668 \\
82.9 & 185.0 & 53.3 & 15613 & 1093 \\
\hline
\end{tabular}

TABLE IX. Velocity derivative moments $\overline{\left\langle(\partial u / \partial x)^{n}\right\rangle} \overline{\left\langle(\partial u / \partial x)^{2}\right\rangle^{n / 2}}, n$ $=4,5, \ldots, 12$ compared with results from $240^{3}$ numerical simulation at $R_{\lambda}=150$ (Vincent and Meneguzzi ${ }^{14}$ ). A: $\alpha=-0.5, \quad \Gamma_{0} / v=100$, $N R^{2} / a=0.6, \phi=0.475$. B: $\alpha=-0.25, \Gamma_{0} / v=200, N R^{2} / a=0.2$, and $\phi$ $=0.775$.

\begin{tabular}{|c|c|c|c|c|}
\hline \multirow[b]{2}{*}{$n$} & \multirow{2}{*}{$\begin{array}{l}\text { Vincent } \\
\text { and } \\
\text { Meneguzzi } \\
\text { No tails }\end{array}$} & \multirow{2}{*}{$\begin{array}{l}\text { Vincent } \\
\text { and } \\
\text { Meneguzzi } \\
\text { Exp. tails }\end{array}$} & \multicolumn{2}{|c|}{ Present } \\
\hline & & & A & B \\
\hline 4 & 5.85 & 6.00 & 6.42 & 6.54 \\
\hline 5 & $-1.01 \times 10^{1}$ & -9.92 & -5.12 & -5.28 \\
\hline 6 & $1.05 \times 10^{2}$ & $1.32 \times 10^{2}$ & $1.22 \times 10^{2}$ & $1.01 \times 10^{2}$ \\
\hline 7 & $-3.89 \times 10^{2}$ & $-3.39 \times 10^{2}$ & $-1.49 \times 10^{2}$ & $-1.25 \times 10^{2}$ \\
\hline 8 & $4.33 \times 10^{3}$ & $9.36 \times 10^{3}$ & $4.13 \times 10^{3}$ & $3.07 \times 10^{3}$ \\
\hline 9 & $-2.69 \times 10^{4}$ & $-1.67 \times 10^{4}$ & $-6.87 \times 10^{3}$ & $-5.17 \times 10^{3}$ \\
\hline 10 & $3.45 \times 10^{5}$ & $1.34 \times 10^{6}$ & $1.80 \times 10^{5}$ & $1.23 \times 10^{5}$ \\
\hline 11 & $-3.11 \times 10^{\circ}$ & $-8.49 \times 10^{5}$ & $-3.8 \times 10^{5}$ & $-2.61 \times 10^{5}$ \\
\hline 12 & $4.61 \times 10^{7}$ & $2.58 \times 10^{8}$ & $9.1 \times 10^{6}$ & $5.8 \times 10^{6}$ \\
\hline
\end{tabular}

and $G_{6}$ at $R_{\lambda}=150$, using the numbers $\hat{G}_{4}=11.0$ and $\hat{G}_{6}=56.8$ from Table VI we obtain $G_{4}=35.3$ and $G_{6}=2434$ compared with results from Vincent and Meneguzzi ${ }^{34}$ $G_{4}=32.8(37.9)$ and $G_{4}=1952(4184)$ where the bracketed numbers indicate results obtained assuming exponential wings.

\section{CONCLUSIONS}

The principal results of the present paper are (99a) and (99b) and (100a) and (100b) in conjunction with (82b) and (84b). It may be observed that the $R_{\lambda}$ dependence displayed in (99a) and (99b) and (100a) and (100b) does not depend on the specific form of the $f$ nor does it depend on the value of the cutoff parameter $\phi$. We suspect that this is true also of the more general Lundgren spiral-vortex solution where additional functions describing the initial conditions are required [e.g., $M$ such functions for Eqs. (1)-(4): see also Lundgren ${ }^{5}$ (Appendix A) where the $f$ functions depend also on $n$ ] although we have provided no relevant proof. The numerical values of $\hat{\Omega}_{2 p}$, $\hat{F}_{2 p}, \hat{G}_{2 p}$, and $\hat{S}_{2 p+1}$ do, however, depend on specific choices for $f$ and $\phi$.

The critical assumptions of the model are that of the constancy of $\Gamma_{0} / v$ independent of the vortex Reynolds number $a^{2} R / 4 v$, and (96) defining the Taylor microscale $\lambda$ in terms of the model parameters $a$ and $R$. These assumptions are in principle subject to quantitative testing by the

TABLE X. $Q_{1}$ and $Q_{2}$ vs $R_{\lambda}$. Left-hand entries are numerical simulation; $128^{3}$, Kerr. ${ }^{9}$ Right-hand entries are present results for $\alpha=-0.5$, $\Gamma_{0} / v=100, N R^{2} / a=0.6$, and $\phi=0.475$.

\begin{tabular}{ccccc}
\hline \hline$R_{\lambda}$ & \multicolumn{2}{c}{$Q_{1}$} & \multicolumn{2}{c}{$Q_{2}$} \\
\hline 28.5 & 4.1 & 4.2 & 4.2 & 5.9 \\
37.2 & 4.2 & 4.5 & 4.3 & 6.4 \\
48.5 & 4.3 & 4.8 & 5.0 & 6.8 \\
55.9 & 4.5 & 5.0 & 5.1 & 7.0 \\
82.9 & 5.0 & 5.5 & 6.0 & 7.8 \\
\hline
\end{tabular}


data of existing and future numerical simulations using, for example, the following test suggested to us by Moore: ${ }^{35}$ consider a cubic box of length $L$ containing the turbulence. Consider the magnitude of the circulation $\Gamma_{\mathscr{H}}=\oint_{\mathscr{R}} \mathbf{u} \cdot d \mathbf{l}$ within all circles of radius $\mathscr{F}$ contained in this box during several or many large-scale eddy turnover times. What is the maximum value of $\Gamma_{\mathscr{K}}$ over $\mathscr{R}$ ?

It was remarked earlier that the Lundgren spectrum (14) and (15) does not contain an $\exp (-B k \eta)$ range as suggested by numerical simulation. One possible explanation may be found in the Townsend-Lundgren model assumption that all vortices feel the same strain $a$. A more realistic expectation is that different vortices may feel different strains, and may have different circulations. Thus among the population of vortex structures, the distribution of these quantities would follow some (unknown) statistics. While some distribution of circulations $\Gamma_{0}$ would not influence the dissipation range, it is clear from the $v k^{2} / a$ form of the exponent in (14), that the statistics of $a$ could alter the dissipation spectrum. If, for example, it is assumed that $a$ is given by a gamma distribution with normalized probability density $p(a)$ given by

$$
p(a)=\frac{q^{q}}{a^{q} \Gamma[q]} a^{q-1} \exp \left(\frac{-q a}{\bar{a}}\right),
$$

where $\bar{a}$ is the average strain felt by a vortex and $q>1$ is some number, then from (14), the spectrum would be of the form

$$
E_{s}(k) \sim k^{q-4 / 3} K_{-q-1 / 3}\left[(8 q v / 3 \bar{a})^{1 / 2} k\right],
$$

where $K_{-q-1 / 3}$ is the $K$-Bessel function. The asymptotic forms of the $K$-Bessel function for small $k$ shows that the inertial range is not modified while the large $k$ asymptotics show that the dissipation range is now of the form $\exp \left[-(8 q v / 3 \bar{a})^{1 / 2} k\right]$.

\section{ACKNOWLEDGMENTS}

The authors wish to thank Dr. T. S. Lundgren, Dr. D. I. Meiron, and Professor D. W. Moore for valuable comments. Thanks are also due to Dr. Maurice Meneguzzi for providing unpublished data on velocity-derivative moments and to Dr. Robert Kerr for providing spectral data obtained from $128^{3}$ numerical simulations.

One of us (PGS) thanks the Department of Energy for support under Grant No. DE-FG03-89ER 25073.

\section{APPENDIX A: THE SPIRAL VORTEX}

In this appendix we give an alternative derivation for Eqs. (1)-(4) describing the evolution of a spiral vortex shear layer. We work in stretched coordinates $(\rho, \theta, \tau)$ of Sec. II A for which the axially symmetric strain is not present. The inviscid limit is the rolling-up of a single vortex sheet, with asymptotic equation

$$
\theta=\Omega(\rho) \tau,
$$

and we note that (A1) can be derived formally from the Birkoff-Rott vortex sheet equation for a nearly circular spiral vortex sheet. The vorticity on the spiral is

$$
\hat{\omega}(\rho, \theta, \tau)=2 \pi f(\rho) \delta[\theta-\Omega(\rho) \tau],
$$

where $f(\rho)$ and $\Omega(\rho)$ are related by (5). The vortex sheet strength, which equals the jump in tangential velocity across the sheet is

$$
\kappa(\rho)=\frac{2 \pi f(\rho)}{\Lambda(\rho) \tau},
$$

where $\Lambda(\rho)=d \Omega / d \rho$.

The effect of viscosity in modifying the vorticity structure can be calculated locally by considering the diffusion of vorticity from a straight vortex sheet whose strength varies in time $\tau$ like (A3) and which lies at a small but finite angle, say $\psi$, to a circle passing through a point on the sheet of radius $\rho$. We first consider the local diffusion process. Let $(x-y)$ be local coordinates in a region surrounding a point on the sheet with extent much smaller than the local sheet radius of curvature $\rho$. Let $x$ be tangential to the sheet that stretches to $\pm \infty$ in the $x$ direction. Since from (A3) $\kappa$ decreases as $\tau^{-1}$, it follows that the sheet must be undergoing stretching in the $(x-y)$ plane. This must be produced locally by a strain field for which we write the local velocity components as $\mathbf{u}_{s}=[\alpha(\tau) x,-\alpha(\tau) y]$ where $\alpha(\tau)$ is the local rate of strain. Let $\hat{\omega}_{0}(y)=\Delta U\left(\tau_{0}\right) \delta(y)$ be the initial vorticity distribution at some time origin $\tau=\tau_{0}$. Then at time $\tau$ the vorticity at distance $y$ from the sheet center is $\left(\mathrm{Kambe}^{36}\right.$ )

$$
\begin{gathered}
\hat{\omega}(y, \tau)=\frac{1}{[4 \pi v T(\tau)]^{1 / 2}} \int_{-\infty}^{\infty} \hat{\omega}_{0}\left(y^{\prime}\right) \\
\quad \times \exp \left(\frac{-\left(A y-y^{\prime}\right)^{2}}{4 v T}\right) d y^{\prime}, \\
A(\tau)=\exp \left(\int_{\tau_{0}}^{\tau} \alpha\left(\tau^{\prime}\right) d \tau^{\prime}\right), \\
T(\tau)=\int_{\tau_{0}}^{\tau} A^{2}\left(\tau^{\prime}\right) d \tau^{\prime} .
\end{gathered}
$$

For large $\tau$, when $\alpha>0$, the asymptotic leading order form of (A4) is

$$
\begin{aligned}
\hat{\omega}(y, \tau)= & \frac{1}{[4 \pi v T(\tau)]^{1 / 2}} \exp \left(\frac{-A^{2} y^{2}}{4 v T}\right) \\
& \times\left[\Delta U\left(\tau_{0}\right)+O\left(\tau^{-1}\right)\right] .
\end{aligned}
$$

The local velocity difference across the diffusing shear layer is found by integrating (A6) with respect to $y$ in $(-\infty, \infty)$, which gives

$$
\Delta U(\tau)=\frac{\Delta U\left(\tau_{0}\right)}{A(\tau)} .
$$

Equating (A7) to (A3) shows that $A$ must be of the form $A(\tau) \sim \mathscr{A}_{0} \tau$ where $\mathscr{H}_{0}$ is a constant. From the second of (A5) it follows that $T(\tau) \sim \mathscr{A}_{0} \tau^{3} / 3$. Using these results in (A6), we obtain to leading order 
$\hat{\omega}(y, \tau)=\frac{\kappa(\tau)}{\left(\frac{4}{3} \pi v \tau\right)^{1 / 2}} \exp \left(-\frac{3 y^{2}}{4 v \tau}\right)$

For a nearly circular vortex sheet the angle $\psi$ is

$\psi \sim 1 / \rho \Lambda \tau$.

Hence the relation between $y$ and $(\rho, \theta, \tau)$ is

$$
y \sim \frac{\theta-\Omega(\rho) \tau}{\Lambda(\rho) \tau}
$$

Using (A10) in (A8) then gives

$$
\hat{\omega}(\rho, \theta, \tau)=\frac{2 \pi f(\rho)}{\Lambda(\rho) \tau\left(\frac{4}{3} \pi v \tau\right)^{1 / 2}} \exp \left(\frac{-3[\theta-\Omega(\rho) \tau]^{2}}{4 v \Lambda^{2}(\rho) \tau^{3}}\right) .
$$

Making use of the relationship

$$
\begin{gathered}
\exp \left(-\beta \Theta^{2}\right) \simeq \sum_{n=-\infty}^{\infty} C_{n} \exp (i n \Theta), \\
C_{n}=\frac{1}{2 \pi} \sqrt{\frac{\pi}{\beta}} \exp \left(\frac{-n^{2}}{4 \beta}\right), \\
\beta^{-1 / 2}<\pi, \text { and choosing }
\end{gathered}
$$

$$
\Theta=\theta-\Omega(\rho) \tau, \quad \beta=\frac{3}{4 v \Lambda^{2}(\rho) \tau^{3}},
$$

then gives, for (A11),

$$
\begin{aligned}
\hat{\omega}(\rho, \theta, \tau)= & f(\rho) \sum_{n=-\infty}^{\infty} \exp (\operatorname{in}[\theta-\Omega(\rho) \tau] \\
& \left.-\frac{n^{2} v \Lambda^{2}(\rho) \tau^{3}}{3}\right)
\end{aligned}
$$

which agrees with (2).

That this analysis is essentially correct may be verified by a direct calculation of the strain rate $\alpha(\tau)$. A point on the vortex sheet feels the principal rates of strain provided by the coarse-grained axisymmetric velocity field with vorticity $f(\rho)+g(\rho)$. A simple calculation shows that this strain is

$$
e_{\rho \theta}=\rho \Lambda(\rho) / 2,
$$

and that the principal axes of strain are at angles $\pi / 4$ to the radius vector from the vortex center. When the strain-rate parallel to the vortex sheet is calculated using (A9) it is found that

$$
\alpha(\tau) \sim \tau^{-1}+\text { higher-order terms }
$$

Equations (A5) and (A16) may be then used to verify the above results.

We comment that the factor of $1 / 3$ in the real part of the exponential in (A14) is produced by the effect of the stretching of the vortex sheet in the $(\rho-\theta)$ plane by the strain rate given by (A16). If this were not present $A$ would equal unity and $T$ from (AS) would be proportional to $\tau$.

\section{APPENDIX B: THE LUNDGREN FILTER}

Suppose we have straight, tubelike structures, distributed randomly in unbounded space, and randomly orientated, with a density $N$, defined as the average number of intersections per unit area. Suppose that relative to a coordinate system fixed in the structure, we have two arbitrary scalar functions which we denote by $f(\mathbf{r}), g(\mathbf{r})$ (not to be confused with $f$ and $g$ used in the main text to specifically denote vorticity distributions) of finite extent, where $\mathbf{r}=\left(r_{1}, r_{2}\right)$ is a two-dimensional vector perpendicular to the axis of a structure. These functions then define stationary random functions of position, which we denote by $\widetilde{f}(\mathbf{x}), \widetilde{g}(\mathbf{x})$, which are the sums of the values of these functions produced by the structures. Note that $\mathbf{x}=\left(x_{1}, x_{2}, x_{3}\right)$ is a three-dimensional vector. We want the ensemble average

$$
\overline{\widetilde{f}(\mathbf{x}) \tilde{g}(\mathbf{x}+\rho)} \equiv F(\rho) .
$$

For the present, we deal with the case when the tubes are distributed isotropically in space. In this case, $F$ depends only on $\rho=|\rho|$. The values of $f$ and $g$ are assumed to be constant along the structure. Then, identifying spatial averages with ensemble averages,

$$
\begin{aligned}
F(\rho)= & \frac{N}{4 \pi} \int_{-\infty}^{\infty} \int_{-\infty}^{\infty} \int_{0}^{\pi} \int_{0}^{2 \pi} f\left(r_{1}, r_{2}\right) \\
& \times g\left(r_{1}+\rho_{1}, r_{2}+\rho_{2}\right) \sin \alpha d r_{1} d r_{2} d \alpha d \beta,
\end{aligned}
$$

where

$$
\rho_{1}=\rho \sin \alpha \cos \beta, \quad \rho_{2}=\rho \sin \alpha \sin \beta,
$$

$\alpha$ and $\beta$ being the latitudinal and longitudinal Euler angles of the axis of the structure relative to the direction of $\rho$. It is assumed here that the structures may overlap and are statistically independent.

\section{Define}

$\hat{f}\left(\kappa_{1}, \kappa_{2}\right)=\frac{1}{4 \pi^{2}} \int_{-\infty}^{\infty} \int_{-\infty}^{\infty} f\left(r_{1}, r_{2}\right) e^{-i \kappa_{1} r_{1}-i \kappa_{2} r_{2}} d r_{1} d r_{2}$,

$$
\hat{g}\left(\kappa_{1}, \kappa_{2}\right)=\frac{1}{4 \pi^{2}} \int_{-\infty}^{\infty} \int_{-\infty}^{\infty} g\left(r_{1}, r_{2}\right) e^{-i \kappa_{1} r_{1}-i \kappa_{2} r_{2}} d r_{1} d r_{2} .
$$

Substituting into Eq. (B2) the inverse formulas

$$
\begin{aligned}
& f\left(r_{1}, r_{2}\right)=\int_{-\infty}^{\infty} \int_{-\infty}^{\infty} \hat{f}\left(\kappa_{1}, \kappa_{2}\right) e^{i \kappa_{1} r_{1}+i \kappa_{2} r_{2}} d \kappa_{1} d \kappa_{2}, \\
& g\left(r_{1}, r_{2}\right)=\int_{-\infty}^{\infty} \int_{-\infty}^{\infty} \hat{g}\left(\kappa_{1}, \kappa_{2}\right) e^{i \kappa_{1} r_{1}+i \kappa_{2} r_{2}} d \kappa_{1} d \kappa_{2},
\end{aligned}
$$

and making repeated use of the result

$$
\int_{-\infty}^{\infty} e^{i \kappa_{1} r_{1}} d r_{1}=2 \pi \delta\left(\kappa_{1}\right), \text { etc., }
$$

where $\delta\left(\kappa_{1}\right)$ is the Dirac delta function, we obtain

$$
\begin{aligned}
F(\rho)= & \pi N \int_{-\infty}^{\infty} \int_{-\infty}^{\infty} \int_{0}^{\pi} \int_{0}^{2 \pi} \hat{f}\left(\kappa_{1}, \kappa_{2}\right) \hat{g}^{*}\left(\kappa_{1}, \kappa_{2}\right) \\
& \times e^{-i \rho_{1} \kappa_{1}-i \rho_{2} \kappa_{2}} \sin \alpha d \kappa_{1} d \kappa_{2} d \alpha d \beta,
\end{aligned}
$$


where $*$ denotes the complex conjugate. We want the power spectrum corresponding to $F(\rho)$ defined as follows:

$$
\begin{aligned}
\hat{F}(k)= & \frac{1}{8 \pi^{3}} \int_{-\infty}^{\infty} \int_{-\infty}^{\infty} \int_{-\infty}^{\infty} F(\rho) \\
& \times e^{-i k_{1} \rho_{1}-i k_{2} \rho_{2}-i k_{3} \rho_{3}} d \rho_{1} d \rho_{2} d \rho_{3}, \\
F(\rho)= & \int_{-\infty}^{\infty} \int_{-\infty}^{\infty} \int_{-\infty}^{\infty} \hat{F}(k) \\
& \times e^{i k_{1} \rho_{1}+i k_{2} \rho_{2}+i k_{3} \rho_{3}} d k_{1} d k_{2} d k_{3} .
\end{aligned}
$$

It is a consequence of the assumed isotropy that $\hat{F}$ depends only on wave number in the combination $k=|\mathbf{k}|$. The power spectrum of $f g$ is

$$
\begin{aligned}
E_{f g}(k) & =\iint \hat{F}(\mathbf{k}) d S(k) \\
& =4 \pi k^{2} \hat{F}(k) \text { when isotropic, }
\end{aligned}
$$

where $d S(k)$ is the element of surface area on the sphere of radius $k$. Note that

$$
F(0)=\int_{0}^{\infty} E_{f g}(k) d k
$$

Integrating Eqs. (B8) over the surface of spheres of radius $\rho$ and $k$, respectively, we obtain

$$
\begin{aligned}
& E_{f g}(k)=\frac{2}{\pi} \int_{0}^{\infty} k \rho \sin k \rho F(\rho) d \rho, \\
& F(\rho)=\int_{0}^{\infty} \frac{\sin k \rho}{k \rho} E_{f g}(k) d k .
\end{aligned}
$$

Substituting Eq. (B7) into the integral for the power spectrum, we obtain

$$
\begin{aligned}
E_{f g}(k)= & 2 N \int_{-\infty}^{\infty} \int_{-\infty}^{\infty} \int_{0}^{\pi} \int_{0}^{2 \pi} \int_{0}^{\infty} \hat{f}\left(\kappa_{1}, \kappa_{2}\right) \\
& \times \hat{g}^{*}\left(\kappa_{1}, \kappa_{2}\right) e^{-i \rho_{1} \kappa_{1}-i \rho_{2} \kappa_{2}} k \rho \\
& \times \sin (k \rho) \sin \alpha d \kappa_{1} d \kappa_{2} d \alpha d \beta d \rho
\end{aligned}
$$

To evaluate this integral, we note that we can change variables and use

$$
\rho^{2} \sin \alpha d \alpha d \beta d \rho=d \rho_{1} d \rho_{2} d \rho_{3},
$$

and

$$
\frac{\sin k \rho}{k \rho}=\frac{1}{4 \pi} \iint e^{i k_{1} \rho_{1}+i k_{2} \rho_{2}+i k_{3} \rho_{3}} \frac{d S(k)}{k^{2}} .
$$

Then Eq. (B12) can be written

$$
\begin{aligned}
E_{f g}(k)= & \frac{N}{2 \pi} \int_{-\infty}^{\infty} \int_{-\infty}^{\infty} \int_{-\infty}^{\infty} \int_{-\infty}^{\infty} \int_{-\infty}^{\infty} \hat{f}\left(\kappa_{1}, \kappa_{2}\right) \hat{g}^{*}\left(\kappa_{1}, \kappa_{2}\right) e^{-i \rho_{1} \kappa_{1}-i \rho_{2} \kappa_{2}} \\
& \times \iint e^{i k_{1} \rho_{1}+i k_{2} \rho_{2}+i k_{3} \rho_{3}} d S(k) d \kappa_{1} d \kappa_{2} d \rho_{1} d \rho_{2} d \rho_{3} \\
= & 4 \pi^{2} N \int_{-\infty}^{\infty} \int_{-\infty}^{\infty} \iint \hat{f}\left(\kappa_{1}, \kappa_{2}\right) \hat{g}^{*}\left(\kappa_{1}, \kappa_{2}\right) \delta\left(k_{1}-\kappa_{1}\right) \delta\left(k_{2}-\kappa_{2}\right) \delta\left(k_{3}\right) d \kappa_{1} d \kappa_{2} d S(k),
\end{aligned}
$$

on use of Eq. (B6). Integrating with respect to $\kappa_{1}, \kappa_{2}$, and $k_{3}$ gives

$$
E_{f g}(k)=4 \pi^{2} N k \oint \hat{f}\left(k_{1}, k_{2}\right) \hat{g}^{*}\left(k_{1}, k_{2}\right) d \theta,
$$

where $k_{1}=k \cos \theta, k_{2}=k \sin \theta$, and $0<\theta<2 \pi$.

Expressing the Fourier transforms in Eqs. (B4) by integrals in polar coordinates gives an expression equivalent to Lundgren's Eq. (58).

We note in passing that a closely similar analysis can be carried out for a purely two-dimensional flow. In this case, Eq. (B2) for the correlation coefficient becomes

$$
\begin{aligned}
F(\rho)= & \frac{N}{\pi} \int_{-\infty}^{\infty} \int_{-\infty}^{\infty} \int_{0}^{2 \pi} f\left(r_{1}, r_{2}\right) g\left(r_{1}+\rho \cos \theta, r_{2}\right. \\
& +\rho \sin \theta) d r_{1} d r_{2} d \theta .
\end{aligned}
$$

Repeating the analysis mutatis mutandis leads to the same equation (B16).

In three dimensions, when $\hat{f}(\mathbf{x})$ and $\hat{g}(\mathbf{x})$ may be components of vectors and tensors, it is necessary to intro- duce integrals over the Euler angles. For example, if $f$ and $g$ are the magnitudes of vectors parallel to the axis of the structure, the correlation coefficient of the product of the one component of $\hat{\mathbf{f}}$ and the two component of $\hat{\mathbf{g}}$ is $F(\rho)$ in Eq. (B7) multiplied by $\left\langle E_{13} E_{23}\right\rangle$, where $E_{i j}$ are the Euler angles of the axis of the structure relative to the fixed coordinates, and the angle brackets denote averages over the angles. In this case, the average for an isotropic distribution is of course zero.

For the vorticity spectrum, we have $\left\langle E_{13}^{2}\right\rangle=\left\langle E_{23}^{2}\right\rangle=\left\langle E_{33}^{2}\right\rangle=1 / 3$, and

$$
\overline{\omega_{1}^{2}}=\frac{1}{3} \overline{\omega^{2}}=\frac{1}{3} \int_{0}^{\infty} \Gamma(k) d k,
$$

where

$$
\Gamma(k)=4 \pi^{2} N k \oint\left|\hat{\omega}\left(k_{1}, k_{2}\right)\right|^{2} d \theta .
$$

The kinetic energy spectrum is $E(k)=\frac{1}{2} \Gamma(k) / k^{2}$. 


\section{APPENDIX C: A COMMENT ON ISOTROPY}

It is well known (e.g., Batchelor) that isotropy of the small scales requires that

$$
\overline{\overline{\left(\frac{\partial u}{\partial x}\right)^{2}}}=\frac{1}{\overline{\left(\frac{\partial v}{\partial x}\right)^{2}}} .
$$

At first sight, this is inconsistent with the expressions (52b) and (55a), when evaluated with (60), according to the following argument. For simplicity, consider first the contribution from the axisymmetric components. From (60), using (59), we have

$$
\overline{e_{1}^{2}}+\overline{e_{2}^{2}}+\overline{e_{3}^{2}}=\overline{\omega_{0}^{2}}+\frac{3 a^{2}}{2}+2 \overline{\left(\frac{u_{0 \theta}^{2}}{r^{2}}-\frac{\omega_{0} u_{0 \theta}}{r}\right)},
$$

where the last term can be shown to be zero because of (59). Then from (52b) and (55a),

$$
\overline{\overline{\left(\frac{\partial u}{\partial x}\right)^{2}}}=\overline{\omega_{0}^{2}}+\frac{a^{2}}{5}, \quad \overline{\left(\frac{\partial v}{\partial x}\right)^{2}}=\overline{\omega^{2}} \overline{\frac{\omega_{0}^{2}}{12}}+\frac{3 a^{2}}{20},
$$

which clearly disagrees with (C1).

The paradox lies in the implicit assumption that $\overline{\omega^{2}}$ $=\omega_{0}^{2}$. We have to recognize that the unbounded structure of the external strain implies a distribution of vorticity at infinity, which should be included in (55a), so that we should write in (C3)

$$
\overline{\omega^{2}}=\overline{\omega_{0}^{2}}+\overline{\omega_{\infty}^{2}} \text {. }
$$

Comparison with (18), or using the formula (Douady et al. ${ }^{15}$ )

$$
\int\left(2 e_{i j}^{2}-\omega^{2}\right) d V=-2 \int \nabla^{2} p d V
$$

where the right-hand side is a divergence, with $e_{i j}$ diagonal $\left(e_{11}=-a / 2, e_{22}=-a / 2, e_{33}=a\right)$, suggests

$$
\overline{\omega_{\infty}^{2}}=3 a^{2} \text {. }
$$

The isotropy relation is then satisfied.

For contributions from the asymmetric spiral, we use (65) and $\overline{\omega^{2}}=\overline{\omega^{\prime 2}}$ to obtain $\overline{e_{1}^{2}}+\overline{e_{2}^{2}}+\overline{e_{3}^{2}}=\frac{1}{2} \overline{\omega^{\prime 2}}$. The expressions (52b) and (55a) then agree with $(\mathrm{Cl})$.

The higher-order moments are dominated by the asymmetric contributions, and no inconsistencies arise.

'A. A. Townsend, "On the fine-scale structure of turbulence," Proc. R. Soc. London Ser. A 208, 534 (1951).

${ }^{2}$ S. Corrsin, "Turbulent dissipation fluctuations," Phys. Fluids 5, 1301 (1962).

${ }^{3}$ A. A. Tennekes, "Simple model for the small-scale structure of turbulence," Phys. Fluids 11, 669 (1968).

${ }^{4}$ P. G. Saffman, "Lectures on homogeneous turbulence," in Topics in Nonlinear Physics, edited by N. J. Zabusky (Springer-Verlag, Berlin, 1968).

${ }^{5}$ T. S. Lundgren, "Strained spiral vortex model for turbulent fine structure," Phys. Fluids 25, 2193 (1982).
${ }^{6} \mathrm{~W} . \mathrm{T}$. Ashurst, "Is turbulence a collection of Burgons?" submitted to Phys. Fluids A.

${ }^{7}$ J. Jiménez, "On small scale vortices in turbulent flows," Center for Turbulence Research Annual Briefs (Stanford U.P., Stanford, 1991).

${ }^{8}$ W. T. Ashurst, A. R. Kerstein, R. M. Kerr, and C. H. Gibson, "Alignment of vorticity and scalar-gradient in simulated Navier-Stokes turbulence," Phys. Fluids 30, 2343 (1987).

${ }^{9}$ R. M. Kerr, "Higher-order derivative correlations and the alignment of small-scale structures in isotropic numerical turbulence," J. Fluid Mech. 153, 31 (1985); see also NASA Tech. Memo. TM 84407 (1983).

${ }^{10}$ R. M. Kerr, "Velocity, scalar and transfer spectra in numerical turbulence," J. Fluid Mech. 211, 309 (1990).

${ }^{11}$ I. Hosokawa and K. Yamamoto, "Intermittency of dissipation in directly simulated fully developed turbulence," J. Phys. Soc. Jpn. 59, 401 (1990).

${ }^{12}$ Z.-S. She, E. Jackson, and S. A. Orszag, "Intermittent vortex structures in homogeneous isotropic turbulence," Nature 344, 226 (1990).

${ }^{13}$ G. R. Ruetsch and M. R. Maxey, "Small-scale features of vorticity and passive scalar fields in homogeneous isotropic turbulence," Phys. Fluids A 3, 1587 (1991).

${ }^{14} \mathrm{~A}$. Vincent and M. Meneguzzi, "The spatial structure and statistical properties of homogeneous turbulence," J. Fluid Mech. 225, 1 (1991).

${ }^{15}$ S. Douady, Y. Couder, and M. E. Brachet, "Direct observation of the intermittency of intense vorticity filaments in turbulence," Phys. Rev. Lett. 67, 983 (1991).

${ }^{16} \mathrm{~J}$. C. McWilliams, "The emergence of isolated coherent vortices in turbulent flow," J. Fluid Mech. 146, 21 (1984).

${ }^{17} \mathrm{~J}$. C. Neu, "The dynamics of stretched vortices," J. Fluid Mech. 143, 2397 (1984).

${ }^{18} \mathrm{~S}$. J. Lin and G. M. Corcos, "The mixing layer: Deterministic models of a turbulent flow. Part 3. The effect of plane strain on the dynamics of streamwise vortices," J. Fluid Mech. 141, 139 (1984).

${ }^{19} \mathrm{C}$. W. Van Atta and R. A. Antonia, "Reynolds number dependence of skewness and flatness factors of turbulent velocity derivatives," Phys. Fluids 23, 252 (1980).

${ }^{20}$ D. W. Moore and P. G. Saffman, "Axial flow in laminar trailing vortices," Proc. R. Soc. London Ser. A 333, 491 (1973).

${ }^{21} \mathrm{D}$. I. Pullin, "On similarity flows containing two-branched vortex sheets," in Mathematical Aspects of Vortex Dynamics, edited by W. Caflisch (SIAM, New York, 1989), pp. 97-106.

${ }^{22}$ J. M. Burgers, "A mathematical model illustrating the theory of turbulence," Adv. Appl. Mech. 1, 171 (1948).

${ }^{23}$ N. Rott, "On the viscous core of a line vortex," Z. Angew. Math. Phys. IXb, 543 (1948)

${ }^{24}$ L. Onsager, "Statistical hydrodynamics," Nuovo Cimento 6, Supplement, 279 (1949).

${ }^{25} \mathrm{G}$. K. Batchelor, The Theory of Homogeneous Turbulence (Cambridge U.P., Cambridge, 1953).

${ }^{26} \mathrm{R}$. Betchov, "An inequality concerning the production of vorticity in isotropic turbulence," J. Fluid Mech. 1, 497 (1956).

${ }^{27}$ G. K. Batchelor and A. A. Townsend, "Turbulent diffusion," in Surveys in Mechanics, edited by G. K. Batchelor and R. M. Davies (Cambridge U.P., Cambridge, 1956).

${ }^{28} \mathrm{~A}$. M. Yaglom, "Laws of small-scale turbulence in atmospheric and ocean," Atmos. Ocean. Phys. 17, 919 (1975).

${ }^{29}$ L. M. Smith and W. C. Reynolds, "The dissipation-range spectrum and the velocity-derivative skewness in turbulent flows," Phys. Fluids A 3, 992 (1991).

${ }^{30}$ D. R. Chapman, "Computational aerodynamics development and outlook," AIAA J. 17, 1293 (1979).

${ }^{31} \mathrm{E}$. D. Siggia, "Invariants for the one-point vorticity and strain-rate correlation functions," Phys. Fluids 24, 1934 (1981).

${ }^{32} \mathrm{H}$. Jeffreys and B. Jeffreys, Methods of Mathematical Physics (Cambridge U.P., Cambridge, 1950).

${ }^{33}$ P. Dimotakis (private communication).

${ }^{34} \mathrm{M}$. Meneguzzi (private communication).

${ }^{35} \mathrm{D}$. W. Moore (private communication).

${ }^{36}$ T. Kambe, "Some dissipation mechanisms in vortex systems," in Turbulence and Chaotic Phenomena in Fluids, edited by T. Tatsumo (Elsevier, New York, 1983), pp. 239-244. 